\title{
ASSOCIATED GRADED ALGEBRAS AND COALGEBRAS
}

\author{
ALESSANDRO ARDIZZONI AND CLAUDIA MENINI
}

\begin{abstract}
We investigate the notion of associated graded coalgebra (algebra) of a bialgebra with respect to a subbialgebra (quotient bialgebra) and characterize those which are bialgebras of type one in the framework of abelian braided monoidal categories.
\end{abstract}

\section{Contents}

Introduction

1. Preliminaries and Notations

2. The Associated Graded Coalgebra

3. The Associated Graded Algebra

4. The Associated Graded Coalgebra of a Bialgebra With Respect to a Subbialgebra

5. The Associated Graded Algebra of a Bialgebra With Respect to a Quotient bialgebra Appendix A. Technicalities

References

\section{INTRODUCTION}

Let $H$ be a braided bialgebra in a cocomplete and complete abelian braided monoidal category $(\mathcal{M}, c)$ satisfying $A B 5$. Assume that the tensor product commutes with direct sums and is twosided exact. Let $M$ be in ${ }_{H}^{H} \mathcal{M}_{H}^{H}$. Let $T=T_{H}(M)$ be the relative tensor algebra and let $T^{c}=$ $T_{H}^{c}(M)$ be the relative cotensor coalgebra as introduced in AMS1. Then both $T$ and $T^{c}$ have a natural structure of graded braided bialgebra and the natural algebra morphism from $T$ to $T^{c}$, which coincide with the canonical injections on $H$ and $M$, is a graded bialgebra homomorphism. Thus its image is a graded braided bialgebra which is denoted by $H[M]$ and called the braided bialgebra of type one associated to $H$ and $M$. Ordinary bialgebras of type one were introduced by Nichols in $\mathbb{N i}$. They came out to play a relevant role in the theory of Hopf Algebras. In particular, their "coinvariant" part, called Nichols algebra, has been deeply investigated, see e.g. [Ro], [AS] and the references therein.

Let $B \hookrightarrow E$ be a monomorphism in $\mathcal{M}$ which is a braided bialgebra homomorphism in $\mathcal{M}$. Under some technical assumptions, we prove in Theorem 4.7 that the following assertions are equivalent.

(1) $g r_{B} E$ is the braided bialgebra of type one associated to $B$ and $\frac{B \wedge_{E} B}{B}$.

(2) $\operatorname{gr}_{B} E$ is strongly $\mathbb{N}$-graded as an algebra (in the sense of Definition 3.2).

(3) $\oplus_{n \in \mathbb{N}} B^{\wedge_{E} n+1}$ is strongly $\mathbb{N}$-graded as an algebra.

(4) $B^{\wedge_{E} n+1}=\left(B \wedge_{E} B\right)^{\cdot{ }^{E} n}$ for every $n \geq 2$.

Here $g r_{B} E$ denotes the associated graded coalgebra $\oplus_{n \in \mathbb{N}} \frac{B^{\wedge} E^{n+1}}{B^{\wedge} E^{n}}$. As an application, in Corollary 4.8, we consider the case of a subbialgebra $H$ of a bialgebra $E$ over a field $K$.

Similar results are obtained in the case $g r_{I} E:=\oplus_{n \in \mathbb{N}} \frac{I^{n}}{I^{n+1}}$ where $I$ is the kernel of an epimorphism $\pi: E \rightarrow B$ which is a braided bialgebra homomorphism in $\mathcal{M}$.

1991 Mathematics Subject Classification. Primary 18D10; Secondary 16W30.

Key words and phrases. Braided bialgebras, Monoidal categories, Cotensor Coalgebras.

This paper was written while the authors were members of G.N.S.A.G.A. with partial financial support from M.I.U.R. within the National Research Project PRIN 2007. 
The paper is organized as follows. In Section 1 we recall some definitions and introduce basic notations needed in the paper. In Section 2, we prove that the associated graded coalgebra $\operatorname{gr}_{C} E:=$ $\oplus_{n \in \mathbb{N}} \frac{C^{\wedge} E^{n+1}}{C^{\wedge} E^{n}}$ for a given subcoalgebra $C$ of a coalgebra $E$ in $\mathcal{M}$, is a strongly $\mathbb{N}$-graded coalgebra (see Theorem 2.10) and hence it can be characterized as in Theorem 2.12. Dual results are obtained in Section 3 for the associated graded algebra $\operatorname{gr}_{I} A:=\oplus_{n \in \mathbb{N}} \frac{I^{n}}{I^{n+1}}$, where $I$ is an ideal of an algebra $A$ in $\mathcal{M}$. In Section 4 , we firstly show that $g r_{B} E$ is a graded braided bialgebra in $(\mathcal{M}, c)$ (see Theorem 4.6) and then prove Theorem 4.7 which is the main result of the paper. Section 5 deals with the dual results. For the reader's sake, some technicalities are collected in Appendix A.

Finally we would like to outline that many results are firstly stated and proved in the coalgebra case where we found the proofs less straightforward. Also proofs in the algebra case are not given whenever they would have been an easy adaptation of the coalgebra ones.

In AM2], results of the present paper are applied to study strictly graded bialgebras.

\section{Preliminaries and Notations}

Notations. Let $\left[\left(X, i_{X}\right)\right]$ be a subobject of an object $E$ in an abelian category $\mathcal{M}$, where $i_{X}=i_{X}^{E}: X \hookrightarrow E$ is a monomorphism and $\left[\left(X, i_{X}\right)\right]$ is the associated equivalence class. By abuse of language, we will say that $\left(X, i_{X}\right)$ is a subobject of $E$ and we will write $\left(X, i_{X}\right)=\left(Y, i_{Y}\right)$ to mean that $\left(Y, i_{Y}\right) \in\left[\left(X, i_{X}\right)\right]$. The same convention applies to cokernels. If $\left(X, i_{X}\right)$ is a subobject of $E$ then we will write $\left(E / X, p_{X}\right)=\operatorname{Coker}\left(i_{X}\right)$, where $p_{X}=p_{X}^{E}: E \rightarrow E / X$.

Let $\left(X_{1}, i_{X_{1}}^{Y_{1}}\right)$ be a subobject of $Y_{1}$ and let $\left(X_{2}, i_{X_{2}}^{Y_{2}}\right)$ be a subobject of $Y_{2}$. Let $x: X_{1} \rightarrow X_{2}$ and $y: Y_{1} \rightarrow Y_{2}$ be morphisms such that $y \circ i_{X_{1}}^{Y_{1}}=i_{X_{2}}^{Y_{2}} \circ x$. Then there exists a unique morphism, which we denote by $y / x=\frac{y}{x}: Y_{1} / X_{1} \rightarrow Y_{2} / X_{2}$, such that $\frac{y}{x} \circ p_{X_{1}}^{Y_{1}}=p_{X_{2}}^{Y_{2}} \circ y$ :

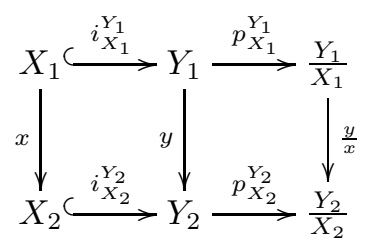

$\delta_{u, v}$ will denote the Kronecker symbol for every $u, v \in \mathbb{N}$.

1.1. Monoidal Categories. Recall that (see [Ka, Chap. XI]) a monoidal category is a category $\mathcal{M}$ endowed with an object $\mathbf{1} \in \mathcal{M}$ (called unit), a functor $\otimes: \mathcal{M} \times \mathcal{M} \rightarrow \mathcal{M}$ (called tensor product), and functorial isomorphisms $a_{X, Y, Z}:(X \otimes Y) \otimes Z \rightarrow X \otimes(Y \otimes Z), l_{X}: \mathbf{1} \otimes X \rightarrow X$, $r_{X}: X \otimes \mathbf{1} \rightarrow X$, for every $X, Y, Z$ in $\mathcal{M}$. The functorial morphism $a$ is called the associativity constraint and satisfies the Pentagon Axiom, that is the following relation

$$
\left(U \otimes a_{V, W, X}\right) \circ a_{U, V \otimes W, X} \circ\left(a_{U, V, W} \otimes X\right)=a_{U, V, W \otimes X} \circ a_{U \otimes V, W, X}
$$

holds true, for every $U, V, W, X$ in $\mathcal{M}$. The morphisms $l$ and $r$ are called the unit constraints and they obey the Triangle Axiom, that is $\left(V \otimes l_{W}\right) \circ a_{V, \mathbf{1}, W}=r_{V} \otimes W$, for every $V, W$ in $\mathcal{M}$.

A braided monoidal category $(\mathcal{M}, c)$ is a monoidal category $(\mathcal{M}, \otimes, \mathbf{1})$ equipped with a braiding $c$, that is a natural isomorphism $c_{X, Y}: X \otimes Y \longrightarrow Y \otimes X$ for every $X, Y, Z$ in $\mathcal{M}$ satisfying

$$
c_{X \otimes Y, Z}=\left(c_{X, Z} \otimes Y\right)\left(X \otimes c_{Y, Z}\right) \quad \text { and } \quad c_{X, Y \otimes Z}=\left(Y \otimes c_{X, Z}\right)\left(c_{X, Y} \otimes Z\right) .
$$

For further details on these topics, we refer to [Ka, Chapter XIII].

It is well known that the Pentagon Axiom completely solves the consistency problem arising out of the possibility of going from $((U \otimes V) \otimes W) \otimes X$ to $U \otimes(V \otimes(W \otimes X))$ in two different ways (see Mj1, page 420]). This allows the notation $X_{1} \otimes \cdots \otimes X_{n}$ forgetting the brackets for any object obtained from $X_{1}, \cdots X_{n}$ using $\otimes$. Also, as a consequence of the coherence theorem, the constraints take care of themselves and can then be omitted in any computation involving morphisms in $\mathcal{M}$.

Thus, for sake of simplicity, from now on, we will omit the associativity constraints.

The notions of algebra, module over an algebra, coalgebra and comodule over a coalgebra can be 
introduced in the general setting of monoidal categories. Given an algebra $A$ in $\mathcal{M}$ on can define the categories ${ }_{A} \mathcal{M}, \mathcal{M}_{A}$ and ${ }_{A} \mathcal{M}_{A}$ of left, right and two-sided modules over $A$ respectively. Similarly, given a coalgebra $C$ in $\mathcal{M}$, one can define the categories of $C$-comodules ${ }^{C} \mathcal{M}, \mathcal{M}^{C},{ }^{C} \mathcal{M}^{C}$. For more details, the reader is referred to [AMS2].

Definitions 1.2. Let $\mathcal{M}$ be a monoidal category.

We say that $\mathcal{M}$ is an abelian monoidal category if $\mathcal{M}$ is abelian and both the functors $X \otimes(-)$ : $\mathcal{M} \rightarrow \mathcal{M}$ and $(-) \otimes X: \mathcal{M} \rightarrow \mathcal{M}$ are additive and right exact, for any $X \in \mathcal{M}$.

We say that $\mathcal{M}$ is an coabelian monoidal category if $\mathcal{M}^{o}$ is an abelian monoidal category, where $\mathcal{M}^{o}$ denotes the dual monoidal category of $\mathcal{M}$. Recall that $\mathcal{M}^{o}$ and $\mathcal{M}$ have the same objects but $\mathcal{M}^{o}(X, Y)=\mathcal{M}(Y, X)$ for any $X, Y$ in $\mathcal{M}$.

Given an algebra $A$ in an abelian monoidal category $\mathcal{M}$, there exist a suitable functor $\otimes_{A}$ : ${ }_{A} \mathcal{M}_{A} \times{ }_{A} \mathcal{M}_{A} \rightarrow{ }_{A} \mathcal{M}_{A}$ and constraints that make the category $\left({ }_{A} \mathcal{M}_{A}, \otimes_{A}, A\right)$ abelian monoidal, see [AMS2, 1.11]. The tensor product over $A$ in $\mathcal{M}$ of a right $A$-module $V$ and a left $A$-module $W$ is defined to be the coequalizer:

$$
(V \otimes A) \otimes W \Longrightarrow V \otimes W \stackrel{{ }^{A} \chi_{V, W}}{\longrightarrow} V \otimes_{A} W \longrightarrow 0
$$

Note that, since $\otimes$ preserves coequalizers, then $V \otimes_{A} W$ is also an $A$-bimodule, whenever $V$ and $W$ are $A$-bimodules.

Dually, let $\mathcal{M}$ be a coabelian monoidal category.

Given a coalgebra $(C, \Delta, \varepsilon)$ in $\mathcal{M}$, there exist of a suitable functor $\square_{C}:{ }^{C} \mathcal{M}^{C} \times{ }^{C} \mathcal{M}^{C} \rightarrow{ }^{C} \mathcal{M}^{C}$ and constraints that make the category $\left({ }^{C} \mathcal{M}^{C}, \square_{C}, C\right)$ coabelian monoidal. The cotensor product over $C$ in $\mathcal{M}$ of a right $C$-bicomodule $V$ and a left $C$-comodule $W$ is defined to be the equalizer:

$$
0 \longrightarrow V \square_{C} W \stackrel{C \varsigma_{V, W}}{\longrightarrow} V \otimes W \rightleftharpoons V \otimes(C \otimes W)
$$

Note that, since $\otimes$ preserves equalizers, then $V \square_{C} W$ is also a $C$-bicomodule, whenever $V$ and $W$ are $C$-bicomodules.

1.3. Graded Objects. Let $\left(X_{n}\right)_{n \in \mathbb{N}}$ be a sequence of objects in a monoidal category $\mathcal{M}$ which is cocomplete abelian and let

$$
X=\bigoplus_{n \in \mathbb{N}} X_{n}
$$

be their coproduct in $\mathcal{M}$. In this case we also say that $X$ is a graded object of $\mathcal{M}$ and that the sequence $\left(X_{n}\right)_{n \in \mathbb{N}}$ defines a graduation on $X$. A morphism

$$
f: X=\bigoplus_{n \in \mathbb{N}} X_{n} \rightarrow Y=\bigoplus_{n \in \mathbb{N}} Y_{n}
$$

is called a graded homomorphism whenever there exists a family of morphisms $\left(f_{n}: X_{n} \rightarrow Y_{n}\right)_{n \in \mathbb{N}}$ such that $f=\oplus_{n \in \mathbb{N}} f_{n}$ i.e. such that

$$
f \circ i_{X_{n}}^{X}=i_{Y_{n}}^{Y} \circ f_{n}, \text { for every } n \in \mathbb{N} .
$$

We fix the following notations:

be the canonical projection and let

$$
p_{n}: X \rightarrow X_{n}
$$

$$
i_{n}: X_{n} \rightarrow X
$$

be the canonical injection for any $n \in \mathbb{N}$.

Given graded objects $X, Y$ in $\mathcal{M}$ we set

$$
(X \otimes Y)_{n}=\oplus_{a+b=n}\left(X_{a} \otimes Y_{b}\right) .
$$

Then this defines a graduation on $X \otimes Y$ whenever the tensor product commutes with direct sums. We denote by

$$
X_{a} \otimes Y_{b} \stackrel{\gamma_{a, b}^{X, Y}}{\rightarrow}(X \otimes Y)_{a+b} \quad \text { and } \quad(X \otimes Y)_{a+b} \stackrel{\omega_{a, Y}^{X, Y}}{\rightarrow} X_{a} \otimes Y_{b}
$$


the canonical injection and projection respectively. We have

$$
\begin{aligned}
\sum_{a+b=n}\left(i_{a}^{X} \otimes i_{b}^{Y}\right) \omega_{a, b}^{X, Y} & =\nabla\left[\left(i_{a}^{X} \otimes i_{b}^{Y}\right)_{a+b=n}\right] \\
\sum_{a+b=n} \gamma_{a, b}^{X, Y}\left(p_{a}^{X} \otimes p_{b}^{Y}\right) & =\Delta\left[\left(p_{a}^{X} \otimes p_{b}^{Y}\right)_{a+b=n}\right]
\end{aligned}
$$

where $\nabla\left[\left(i_{a}^{X} \otimes i_{b}^{Y}\right)_{a+b=n}\right]$ denotes the codiagonal morphism associated to the family $\left(i_{a}^{X} \otimes i_{b}^{Y}\right)_{a+b=n}$ and $\Delta\left[\left(p_{a}^{X} \otimes p_{b}^{Y}\right)_{a+b=n}\right]$ denotes the diagonal morphism associated to the family $\left(p_{a}^{X} \otimes p_{b}^{Y}\right)_{a+b=n}$.

\section{The Associated Graded Coalgebra}

2.1. Let $\mathcal{M}$ be a coabelian monoidal category such that the tensor product commutes with direct sums.

Recall that a graded coalgebra in $\mathcal{M}$ is a coalgebra $(C, \Delta, \varepsilon)$ where

$$
C=\oplus_{n \in \mathbb{N}} C_{n}
$$

is a graded object of $\mathcal{M}$ such that $\Delta: C \rightarrow C \otimes C$ is a graded homomorphism i.e. there exists a family $\left(\Delta_{n}\right)_{n \in \mathbb{N}}$ of morphisms

$$
\Delta_{n}^{C}=\Delta_{n}: C_{n} \rightarrow(C \otimes C)_{n}=\oplus_{a+b=n}\left(C_{a} \otimes C_{b}\right) \text { such that } \Delta=\oplus_{n \in \mathbb{N}} \Delta_{n}
$$

We set

$$
\Delta_{a, b}^{C}=\Delta_{a, b}:=\left(C_{a+b} \stackrel{\Delta_{a+b}}{\rightarrow}(C \otimes C)_{a+b} \stackrel{\omega_{a, b}^{C, C}}{\rightarrow} C_{a} \otimes C_{b}\right) .
$$

A homomorphism $f:\left(C, \Delta_{C}, \varepsilon_{C}\right) \rightarrow\left(D, \Delta_{D}, \varepsilon_{D}\right)$ of coalgebras is a graded coalgebra homomorphism if it is a graded homomorphism too.

Definition 2.2. Let $\left(C=\oplus_{n \in \mathbb{N}} C_{n}, \Delta, \varepsilon\right)$ be a graded coalgebra in $\mathcal{M}$. In analogy with the group graded case (see [NT]), we say that $C$ is a strongly $\mathbb{N}$-graded coalgebra whenever

$\Delta_{i, j}^{C}: C_{i+j} \rightarrow C_{i} \otimes C_{j}$ is a monomorphism for every $i, j \in \mathbb{N}$,

where $\Delta_{i, j}^{C}$ is the morphism defined in Definition 2.1.

Proposition 2.3. AM1, Propositions 2.5 and 2.3] Let $\mathcal{M}$ be a coabelian monoidal category such that the tensor product commutes with direct sums.

1) Let $C=\oplus_{n \in \mathbb{N}} C_{n}$ be a graded object of $\mathcal{M}$ such that there exists a family $\left(\Delta_{a, b}^{C}\right)_{a, b \in \mathbb{N}}$

$$
\Delta_{a, b}^{C}: C_{a+b} \rightarrow C_{a} \otimes C_{b}
$$

of morphisms and a morphism $\varepsilon_{0}^{C}: C_{0} \rightarrow \mathbf{1}$ which satisfy

$$
\begin{gathered}
\left(\Delta_{a, b}^{C} \otimes C_{c}\right) \circ \Delta_{a+b, c}^{C}=\left(C_{a} \otimes \Delta_{b, c}^{C}\right) \circ \Delta_{a, b+c}^{C}, \\
\left(C_{d} \otimes \varepsilon_{0}^{C}\right) \circ \Delta_{d, 0}^{C}=r_{C_{d}}^{-1}, \quad\left(\varepsilon_{0}^{C} \otimes C_{d}\right) \circ \Delta_{0, d}^{C}=l_{C_{d}}^{-1},
\end{gathered}
$$

for every $a, b, c \in \mathbb{N}$. Then there exists a unique morphism $\Delta_{C}: C \rightarrow C \otimes C$ such that

$$
\left(p_{a}^{C} \otimes p_{b}^{C}\right) \circ \Delta_{C}=\Delta_{a, b}^{C} \circ p_{a+b}^{C}, \text { for every } a, b \in \mathbb{N}
$$

holds. Moreover $\left(C=\oplus_{n \in \mathbb{N}} C_{n}, \Delta_{C}, \varepsilon_{C}=\varepsilon_{0}^{C} p_{0}^{C}\right)$ is a graded coalgebra.

2) If $C$ is a graded coalgebra, then

$$
\Delta_{C} \circ i_{n}^{C}=\sum_{a+b=n}\left(i_{a}^{C} \otimes i_{b}^{C}\right) \circ \Delta_{a, b}^{C}
$$

holds, $\varepsilon_{C}=\varepsilon_{C} i_{0}^{C} p_{0}^{C}$ so that $\varepsilon_{C}$ is a graded homomorphism, and we have that (3) and (4) hold for every $a, b, c \in \mathbb{N}$, where $\varepsilon_{0}^{C}=\varepsilon_{C} i_{0}$.

Moreover $\left(C_{0}, \Delta_{0}=\Delta_{0,0}^{C}, \varepsilon_{0}^{C}=\varepsilon_{C} i_{0}^{C}\right)$ is a coalgebra in $\mathcal{M}, i_{0}^{C}$ is a coalgebra homomorphism and, for every $n \in \mathbb{N},\left(C_{n}, \Delta_{0, n}^{C}, \Delta_{n, 0}^{C}\right)$ is a $C_{0}$-bicomodule such that $p_{n}^{C}: C \rightarrow C_{n}$ is a morphism of $C_{0}$-bicomodules ( $C$ is a $C_{0}$-bicomodule through $p_{0}^{C}$ ). 
LEMMA 2.4. Let $\mathcal{M}$ be a coabelian monoidal category such that the tensor product commutes with direct sums.

Let $\left(\left(C_{a}\right)_{a \in \mathbb{N}},\left(\beta_{C_{a}}^{C_{b}}\right)_{a, b \in \mathbb{N}}\right)$ be a direct system in $\mathcal{M}$, where, for $a \leq b, \beta_{C_{a}}^{C_{b}}: C_{a} \rightarrow C_{b}$ is an epimorphism. Assume that there exists a family $\left(\Delta_{a, b}^{C}\right)_{a, b \in \mathbb{N}}$

$$
\Delta_{a, b}^{C}: C_{a+b} \rightarrow C_{a} \otimes C_{b},
$$

of morphisms and a morphism $\varepsilon_{0}^{C}: C_{0} \rightarrow \mathbf{1}$ which satisfy (3), (四),

(7) $\left(\beta_{C_{a}}^{C_{a+1}} \otimes C_{b}\right) \circ \Delta_{a, b}^{C}=\Delta_{a+1, b}^{C} \circ \beta_{C_{a+b}}^{C_{a+b+1}} \quad$ and $\quad\left(C_{a} \otimes \beta_{C_{b}}^{C_{b+1}}\right) \circ \Delta_{a, b}^{C}=\Delta_{a, b+1}^{C} \circ \beta_{C_{a+b}}^{C_{a+b+1}}$

for every $a, b, c \in \mathbb{N}$. Set $C_{-1}:=0$.

Let $\left(E_{n}, i_{E_{n}}^{C_{n}}\right):=\operatorname{ker}\left(\beta_{C_{n}}^{C_{n+1}}\right)$ for every $n \in \mathbb{N}$. that

Then $C=\oplus_{n \in \mathbb{N}} C_{n}$ is a graded coalgebra, there is a unique coalgebra structure on $\oplus_{n \in \mathbb{N}} E_{n}$ such

is a coalgebra homomorphism and

$$
\oplus_{n \in \mathbb{N}} i_{E_{n}}^{C_{n}}: \oplus_{n \in \mathbb{N}} E_{n} \rightarrow \oplus_{n \in \mathbb{N}} C_{n}
$$

1) $E=\oplus_{n \in \mathbb{N}} E_{n}$ is a graded coalgebra such that $\oplus_{n \in \mathbb{N}} i_{E_{n}}^{C_{n}}$ is a graded homomorphism;

2) $\left(i_{E_{a}}^{C_{a}} \otimes i_{E_{b}}^{C_{b}}\right) \circ \Delta_{a, b}^{E}=\Delta_{a, b}^{C} \circ i_{E_{a+b}}^{C_{a+b}}$

3) $\varepsilon_{E}=\varepsilon_{0}^{C} \circ i_{E_{0}}^{C_{0}} \circ p_{0}^{E}$.

Proof. By Proposition 2.3, there exists a unique morphism $\Delta_{C}: C \rightarrow C \otimes C$ such that (6) holds. Moreover $\left(C=\oplus_{n \in \mathbb{N}} C_{n}, \Delta_{C}, \varepsilon_{C}=\varepsilon_{0}^{C} p_{0}^{C}\right)$ is a graded coalgebra.

By left exactness of the tensor functors, we have the exact sequence

$$
0 \rightarrow E_{a} \otimes C_{b} \stackrel{i_{E_{a}}^{C_{a}} \otimes C_{b}}{\longrightarrow} C_{a} \otimes C_{b} \stackrel{\beta_{C a}^{C_{a+1}} \otimes C_{b}}{\longrightarrow} C_{a+1} \otimes C_{b} .
$$

From

$$
\left(\beta_{C_{a}}^{C_{a+1}} \otimes C_{b}\right) \circ \Delta_{a, b}^{C} \circ i_{E_{a+b}}^{C_{a+b}} \underline{\text { 国 }} \Delta_{a+1, b}^{C} \circ \beta_{C_{a+b}}^{C_{a+b+1}} \circ i_{E_{a+b}}^{C_{a+b}}=0 .
$$

and, by exactness of (8), there is a unique morphism $\alpha_{a, b}: E_{a+b} \rightarrow E_{a} \otimes C_{b}$ such that

$$
\left(i_{E_{a}}^{C_{a}} \otimes C_{b}\right) \circ \alpha_{a, b}=\Delta_{a, b}^{C} \circ i_{E_{a+b}}^{C_{a+b}} .
$$

By left exactness of the tensor functors, we have the exact sequence

$$
0 \rightarrow E_{a} \otimes E_{b} \stackrel{E_{a} \otimes i_{E_{b}}^{C_{b}}}{\longrightarrow} E_{a} \otimes C_{b} \stackrel{E_{a} \otimes \beta_{C_{b}}^{C_{b+1}}}{\longrightarrow} E_{a} \otimes C_{b+1} .
$$

We obtain

$$
\begin{aligned}
\left(i_{E_{a}}^{C_{a}} \otimes C_{b+1}\right) \circ\left(E_{a} \otimes \beta_{C_{b}}^{C_{b+1}}\right) \circ \alpha_{a, b} & =\left(C_{a} \otimes \beta_{C_{b}}^{C_{b+1}}\right) \circ\left(i_{E_{a}}^{C_{a}} \otimes C_{b}\right) \circ \alpha_{a, b} \\
& =\left(C_{a} \otimes \beta_{C_{b}}^{C_{b+1}}\right) \circ \Delta_{a, b}^{C} \circ i_{E_{a+b}}^{C_{a+b}}=0
\end{aligned}
$$

where the last equality is analogue to (9). Since $i_{E_{a}}^{C_{a}} \otimes C_{b+1}$ is a monomorphism, we deduce that $\left(E_{a} \otimes \beta_{C_{b}}^{C_{b+1}}\right) \circ \alpha_{a, b}=0$ so that, by exactness of (10), there is a unique morphism $\Delta_{a, b}^{E}: E_{a+b} \rightarrow$ $E_{a} \otimes E_{b}$, such that

We compute

$$
\left(E_{a} \otimes i_{E_{b}}^{C_{b}}\right) \circ \Delta_{a, b}^{E}=\alpha_{a, b}
$$

$$
\left(i_{E_{a}}^{C_{a}} \otimes i_{E_{b}}^{C_{b}}\right) \circ \Delta_{a, b}^{E}=\left(i_{E_{a}}^{C_{a}} \otimes C_{b}\right) \circ\left(E_{a} \otimes i_{E_{b}}^{C_{b}}\right) \circ \Delta_{a, b}^{E}=\left(i_{E_{a}}^{C_{a}} \otimes C_{b}\right) \circ \alpha_{a, b}=\Delta_{a, b}^{C} \circ i_{E_{a+b}}^{C_{a+b}}
$$

so that 2) holds true.

Let us prove that $\Delta_{a, b}^{E}$ fulfills (3). By 2), we infer

$$
\left(i_{E_{a}}^{C_{a}} \otimes i_{E_{b}}^{C_{b}} \otimes i_{E_{c}}^{C_{c}}\right) \circ\left(\Delta_{a, b}^{E} \otimes E_{c}\right) \circ \Delta_{a+b, c}^{E}=\left(\Delta_{a, b}^{C} \otimes C_{c}\right) \circ \Delta_{a+b, c}^{C} \circ i_{E_{a+b+c}}^{C_{a+b+c}},
$$




$$
\left(i_{E_{a}}^{C_{a}} \otimes i_{E_{b}}^{C_{b}} \otimes i_{E_{c}}^{C_{c}}\right) \circ\left(E_{a} \otimes \Delta_{b, c}^{E}\right) \circ \Delta_{a, b+c}^{E}=\left(C_{a} \otimes \Delta_{b, c}^{C}\right) \circ \Delta_{a, b+c}^{C} \circ i_{E_{a+b+c}}^{C_{a+b+c}} .
$$

Since $C$ fulfills (3) and since $i_{E_{a}}^{C_{a}} \otimes i_{E_{b}}^{C_{b}} \otimes i_{E_{c}}^{C_{c}}$ is a monomorphism, we get (3) for $E$.

Let $\varepsilon_{0}^{E}:=\varepsilon_{0}^{C} \circ i_{E_{0}}^{C_{0}}$. Let us prove that (4) hold for $E$. By 2), we have

$$
\begin{aligned}
\left(i_{E_{d}}^{C_{d}} \otimes \mathbf{1}\right) \circ\left(E_{d} \otimes \varepsilon_{0}^{E}\right) \circ \Delta_{d, 0}^{E} & =\left(C_{d} \otimes \varepsilon_{0}^{C}\right) \circ\left(i_{E_{d}}^{C_{d}} \otimes i_{E_{0}}^{C_{0}}\right) \circ \Delta_{d, 0}^{E}=\left(C_{d} \otimes \varepsilon_{0}^{C}\right) \circ \Delta_{d, 0}^{C} \circ i_{E_{d}}^{C_{d}}, \\
\left(i_{E_{d}}^{C_{d}} \otimes \mathbf{1}\right) \circ r_{E_{d}}^{-1} & =r_{C_{d}}^{-1} \circ i_{E_{d}}^{C_{d}} .
\end{aligned}
$$

By (4) for $C$ and since $i_{E_{d}}^{C_{d}} \otimes \mathbf{1}$ is a monomorphism, we get the left equation of (4) for $E$.

Similarly one gets the other equation. Thus, by applying Proposition 2.3, we conclude that $E$ is a graded coalgebra and 3) holds true.

It remains to prove that $i:=\oplus_{n \in \mathbb{N}} i_{E_{n}}^{C_{n}}$ is a coalgebra homomorphism. For every $a, b \in \mathbb{N}$, we have

$$
\begin{aligned}
& (i \otimes i) \circ \Delta_{E} \circ i_{n}^{E} \underline{\underline{\Phi}}(i \otimes i) \circ \sum_{a+b=n}\left(i_{a}^{E} \otimes i_{b}^{E}\right) \circ \Delta_{a, b}^{E} \\
= & \sum_{a+b=n}\left(i_{a}^{C} \otimes i_{b}^{C}\right) \circ\left(i_{E_{a}}^{C_{a}} \otimes i_{E_{b}}^{C_{b}}\right) \circ \Delta_{a, b}^{E} \stackrel{2)}{=} \sum_{a+b=n}\left(i_{a}^{C} \otimes i_{b}^{C}\right) \circ \Delta_{a, b}^{C} \circ i_{E_{a+b}}^{C_{a+b}} \\
& \stackrel{\left(\text { G) } \Delta_{C} \circ i_{n}^{C} \circ i_{E_{n}}^{C_{n}}=\Delta_{C} \circ i \circ i_{n}^{E}\right.}{ }
\end{aligned}
$$

and

$$
\varepsilon_{C} \circ i \circ i_{n}^{E}=\varepsilon_{C} \circ i_{n}^{C} \circ i_{E_{n}}^{C_{n}}=\varepsilon_{0}^{C} \circ p_{0}^{C} \circ i_{n}^{C} \circ i_{E_{n}}^{C_{n}}=\delta_{n, 0} \varepsilon_{0}^{C} \circ i_{E_{0}}^{C_{0}}=\varepsilon_{0}^{C} \circ i_{E_{0}}^{C_{0}} \circ p_{0}^{E} \circ i_{n}^{E}=\varepsilon_{E} \circ i_{n}^{E}
$$

so that $(i \otimes i) \circ \Delta_{E}=\Delta_{C} \circ i$ and $\varepsilon_{C} \circ i=\varepsilon_{E}$. Thus $i$ is a coalgebra homomorphism.

LEMMA 2.5. Let $\mathcal{M}$ be a coabelian monoidal category such that the tensor product commutes with direct sums.

Let $\left(\left(C_{a}\right)_{a \in \mathbb{N}},\left(\beta_{C_{a}}^{C_{b}}\right)_{a, b \in \mathbb{N}}\right)$ be an inverse system in $\mathcal{M}$, where, for $a \leq b, \beta_{C_{b}}^{C_{a}}: C_{b} \rightarrow C_{a}$ is an epimorphism. Assume that there exists a family $\left(\Delta_{a, b}^{C}\right)_{a, b \in \mathbb{N}}$

$$
\Delta_{a, b}^{C}: C_{a+b} \rightarrow C_{a} \otimes C_{b},
$$

of morphisms and a morphism $\varepsilon_{0}^{C}: C_{0} \rightarrow \mathbf{1}$ which satisfy (旬), (4),

$$
\left(\beta_{C_{a+1}}^{C_{a}} \otimes C_{b}\right) \circ \Delta_{a+1, b}^{C}=\Delta_{a, b}^{C} \circ \beta_{C_{a+b+1}}^{C_{a+b}} \quad \text { and } \quad\left(C_{a} \otimes \beta_{C_{b+1}}^{C_{b}}\right) \circ \Delta_{a, b+1}^{C}=\Delta_{a, b}^{C} \circ \beta_{C_{a+b+1}}^{C_{a+b}}
$$

for every $a, b, c \in \mathbb{N}$. Set $C_{-1}:=0$.

Let $\left(E_{n}, i_{E_{n}}^{C_{n}}\right):=\operatorname{ker}\left(\beta_{C_{n}}^{C_{n-1}}\right)$ for every $n \in \mathbb{N}$.

Then $C=\oplus_{n \in \mathbb{N}} C_{n}$ is a graded coalgebra, there is a unique coalgebra structure on $\oplus_{n \in \mathbb{N}} E_{n}$ such that

$$
\oplus_{n \in \mathbb{N}} i_{E_{n}}^{C_{n}}: \oplus_{n \in \mathbb{N}} E_{n} \rightarrow \oplus_{n \in \mathbb{N}} C_{n}
$$

is a coalgebra homomorphism and

1) $E=\oplus_{n \in \mathbb{N}} E_{n}$ is a graded coalgebra such that $\oplus_{n \in \mathbb{N}} i_{E_{n}}^{C_{n}}$ is a graded homomorphism;

2) $\left(i_{E_{a}}^{C_{a}} \otimes i_{E_{b}}^{C_{b}}\right) \circ \Delta_{a, b}^{E}=\Delta_{a, b}^{C} \circ i_{E_{a+b}}^{C_{a+b}}$;

3) $\varepsilon_{E}=\varepsilon_{0}^{C} \circ i_{E_{0}}^{C_{0}} \circ p_{0}^{E}$.

Proof. It is similar to that of Lemma 2.4. 
LEMMA 2.6. Consider the following commutative diagram in an abelian category $\mathcal{C}$.

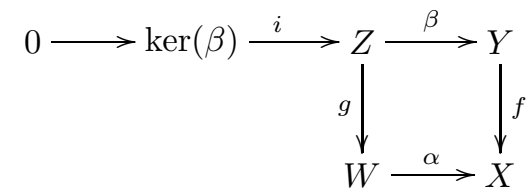

Assume that both $f$ and $g \circ i$ are monomorphisms. Then $g$ is a monomorphism.

Proof. Let $\xi: T \rightarrow Z$ be a morphism such that $g \circ \xi=0$. Then $f \circ \beta \circ \xi=\alpha \circ g \circ \xi=0$ so that, since $f$ is a monomorphism, we get $\beta \circ \xi=0$. By the universal property of kernels $\xi$ factors to a $\operatorname{map} \bar{\xi}: T \rightarrow \operatorname{ker}(\beta)$ such that $i \circ \bar{\xi}=\xi$.

Now $g \circ i \circ \bar{\xi}=g \circ \xi=0$ so that, since $g \circ i$ is a monomorphism, we get $\bar{\xi}=0$.

THEOREM 2.7. With hypothesis and notations of Lemma 2.5, the following assertions are equivalent.

(1) $C=\oplus_{n \in \mathbb{N}} C_{n}$ is a strongly $\mathbb{N}$-graded coalgebra.

(2) $E=\oplus_{n \in \mathbb{N}} E_{n}$ is a strongly $\mathbb{N}$-graded coalgebra.

Proof. Let $\left(\left(C_{a}\right)_{a \in \mathbb{N}},\left(\beta_{C_{a}}^{C_{b}}\right)_{a, b \in \mathbb{N}}\right)$ be an inverse system in $\mathcal{M}$, where, for $a \leq b, \beta_{C_{b}}^{C_{a}}: C_{b} \rightarrow C_{a}$ is an epimorphism. Assume that there exists a family $\left(\Delta_{a, b}^{C}\right)_{a, b \in \mathbb{N}}$

$$
\Delta_{a, b}^{C}: C_{a+b} \rightarrow C_{a} \otimes C_{b}
$$

of morphisms and a morphism $\varepsilon_{0}^{C}: C_{0} \rightarrow \mathbf{1}$ which satisfy (3), (4) and (11). Let $\left(E_{n}, i_{E_{n}}^{C_{n}}\right):=$ $\operatorname{ker}\left(\beta_{C_{n}}^{C_{n-1}}\right)$ for every $n \in \mathbb{N}$.

$(1) \Rightarrow(2)$ It follows from $\left(i_{E_{a}}^{C_{a}} \otimes i_{E_{b}}^{C_{b}}\right) \circ \Delta_{a, b}^{E}=\Delta_{a, b}^{C} \circ i_{E_{a+b}}^{C_{a+b}}$ which holds in view of Lemma 2.5.

$(2) \Rightarrow(1)$ By assumption, $\Delta_{a, b}^{E}$ is a monomorphism for every $a, b \in \mathbb{N}$.

In view of $\left[\mathrm{AM} 1\right.$, Theorem 2.22], it is enough to prove that $\Delta_{a, 1}^{C}: C_{a+1} \rightarrow C_{a} \otimes C_{1}$ is a monomorphism by induction on $a \in \mathbb{N}$.

$a=0)$ From

$$
\left(\varepsilon_{0} \otimes C_{1}\right) \circ \Delta_{0,1}^{C} \stackrel{\text { (4) }}{=} l_{C_{1}}^{-1}
$$

we deduce that $\Delta_{0,1}^{C}$ is a monomorphism.

$a-1 \Rightarrow a)$ Since $\Delta_{a, 1}^{E}$ and $i_{E_{a}}^{C_{a}} \otimes i_{E_{1}}^{C_{1}}$ are monomorphisms and

$$
\left(i_{E_{a}}^{C_{a}} \otimes i_{E_{1}}^{C_{1}}\right) \circ \Delta_{a, 1}^{E}=\Delta_{a, 1}^{C} \circ i_{E_{a+1}}^{C_{a+1}}
$$

which holds in view of Lemma 2.5, we deduce that $\Delta_{a, 1}^{C} \circ i_{E_{a+1}}^{C_{a+1}}$ is a monomorphism too. From (11), we get

$$
\left(\beta_{C_{a}}^{C_{a-1}} \otimes C_{1}\right) \circ \Delta_{a, 1}^{C}=\Delta_{a-1,1}^{C} \circ \beta_{C_{a+1}}^{C_{a}}
$$

Hence by inductive hypothesis, we can apply Lemma 2.6 to the following commutative diagram:

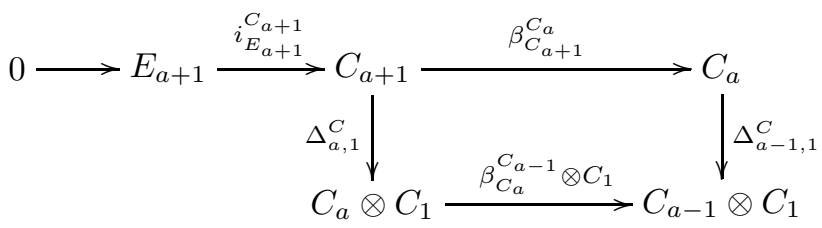

2.8. Let $\mathcal{M}$ be a coabelian monoidal category.

Let $\left(C, i_{C}^{E}\right)$ and $\left(D, i_{D}^{E}\right)$ be two subobjects of a coalgebra $(E, \Delta, \varepsilon)$. Set

$$
\Delta_{C, D}:=\left(p_{C}^{E} \otimes p_{D}^{E}\right) \Delta: E \rightarrow \frac{E}{C} \otimes \frac{E}{D}
$$




$$
\begin{gathered}
\left(C \wedge_{E} D, i_{C \wedge_{E} D}^{E}\right)=\operatorname{ker}\left(\Delta_{C, D}\right), \quad i_{C \wedge_{E} D}^{E}: C \wedge_{E} D \rightarrow E \\
\left(\frac{E}{C \wedge_{E} D}, p_{C \wedge_{E} D}^{E}\right)=\operatorname{Coker}\left(i_{C \wedge_{E} D}^{E}\right)=\operatorname{Im}\left(\Delta_{C, D}\right), \quad p_{C \wedge_{E} D}^{E}: E \rightarrow \frac{E}{C \wedge_{E} D}
\end{gathered}
$$

Moreover, we have the following exact sequence:

$$
0 \longrightarrow C \wedge_{E} D \stackrel{i_{C \wedge_{E} D}^{E}}{\longrightarrow} E \stackrel{p_{C \wedge_{E} D}^{E}}{\longrightarrow} \frac{E}{C \wedge_{E} D} \longrightarrow 0
$$

Since $\left(\frac{E}{C \wedge_{E} D}, p p_{C \wedge_{E} D}^{E}\right)=\operatorname{Coker}\left(i_{C \wedge_{E} D}^{E}\right)$ and $\Delta_{C, D} \circ i_{C \wedge_{E} D}^{E}=0$, by the universal property of the cokernel, there is a unique morphism $\bar{\Delta}_{C, D}: \frac{E}{C \wedge_{E} D} \rightarrow \frac{E}{C} \otimes \frac{E}{D}$ such that the following diagram

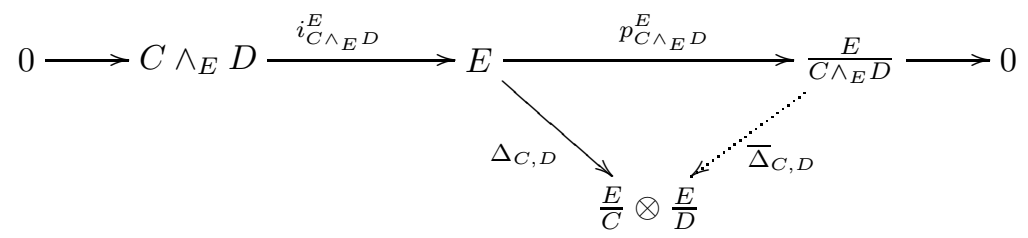

is commutative. Since $\left(\frac{E}{C \wedge_{E} D}, p_{C \wedge_{E} D}^{E}\right)=\operatorname{Im}\left(\Delta_{C, D}\right)$, it comes out that $\bar{\Delta}_{C, D}$ is a monomorphism. Assume now that $\left(C, i_{C}^{E}\right)$ and $\left(D, i_{D}^{E}\right)$ are two subcoalgebras of $(E, \Delta, \varepsilon)$. Since $\Delta_{C, D} \in{ }^{E} \mathcal{M}^{E}$, it is straightforward to prove that $C \wedge_{E} D$ is a coalgebra and that $i_{C \wedge_{E} D}^{E}$ is a coalgebra homomorphism. Consider the case $C=0$.

Since $p_{D}^{E}$ is a morphism in ${ }^{E} \mathcal{M}$, we have

$$
\Delta_{0, D}=\left(\operatorname{Id}_{E} \otimes p_{D}^{E}\right) \circ \Delta=\rho_{E / D}^{l} \circ p_{D}^{E}
$$

Since $\rho_{E / D}^{l}$ is a monomorphism, we deduce that

$$
\left(0 \wedge_{E} D, i_{0 \wedge_{E} D}^{E}\right)=\operatorname{ker}\left(\Delta_{0, D}\right)=\operatorname{ker}\left(p_{D}^{E}\right)=\left(D, i_{D}^{E}\right) .
$$

Analogously, in the case $D=0$, one has

$$
\left(C \wedge_{E} 0, i_{C \wedge_{E} 0}^{E}\right)=\left(C, i_{C}^{E}\right) .
$$

2.9. Let $\left(C, i_{C}^{E}\right)$ be a subobject of a coalgebra $(E, \Delta, \varepsilon)$ in a coabelian monoidal category $\mathcal{M}$. We can define (see AMS2] the $n$-th wedge product $\left(C^{\wedge_{E} n}, i_{C^{\wedge} E^{n}}^{E}\right)$ of $C$ in $E$ where $i_{C^{\wedge} E^{n}}^{E}: C^{\wedge_{E} n} \rightarrow E$. By definition, we have

$$
C^{\wedge_{E} 0}=0 \quad \text { and } \quad C^{\wedge_{E} n}=C^{\wedge_{E}{ }^{n-1}} \wedge_{E} C, \text { for every } n \geq 1 .
$$

One can check that $\left(\left(C \wedge_{E} D\right) \wedge_{E} F, i_{\left(C \wedge_{E} D\right) \wedge_{E} F}^{E}\right)$ and $\left(C \wedge_{E}\left(D \wedge_{E} F\right), i{ }_{C \wedge_{E}\left(D \wedge_{E} F\right)}^{E}\right)$ are isomorphic, for every subobject $C, D, F$ of $E$, and thus can be identified. Therefore $C^{\wedge_{E} i} \wedge_{E} C^{\wedge_{E} j}=$ $C^{\wedge_{E} i+j}$ and we can consider

$$
\bar{\Delta}_{C^{\wedge E^{i}, C^{{ } E^{j}}}}: \frac{E}{C^{\wedge_{E} i+j}} \rightarrow \frac{E}{C^{\wedge_{E} i}} \otimes \frac{E}{C^{\wedge_{E} j}} .
$$

Assume now that $\left(C, i_{C}^{E}\right)$ is a subcoalgebra of the coalgebra $(E, \Delta, \varepsilon)$. Then there is a (unique) coalgebra homomorphism

$$
i_{C^{\wedge_{E}}}^{\wedge^{n+1}}: C^{\wedge_{E} n} \rightarrow C^{\wedge_{E} n+1} \text {, for every } n \in \mathbb{N} .
$$

such that $i_{C^{\wedge} E^{n+1}}^{E} \circ i_{C^{\wedge} E^{n}}^{C^{\wedge} E^{n+1}}=i_{C^{\wedge} E^{n}}^{E}$. We set

and

$$
E_{C}:=\oplus_{n \in \mathbb{N}} \frac{E}{C^{\wedge}{ }_{E} n}
$$

$$
\Delta_{i, j}^{E_{C}}:=\bar{\Delta}_{C^{\wedge} E^{i}, C^{\wedge} E^{j}}: \frac{E}{C^{\wedge_{E} i+j}} \rightarrow \frac{E}{C^{\wedge_{E} i}} \otimes \frac{E}{C^{\wedge_{E} j}}
$$

Since $\Delta$ is coassociative and by definition of $\Delta_{i, j}^{E_{C}}$, it is straightforward to prove that $\Delta_{i, j}^{E_{C}}$ fulfills

$$
\left(\Delta_{a, b}^{E_{C}} \otimes \frac{E}{C^{\wedge_{E} c}}\right) \Delta_{a+b, c}^{E_{C}}=\left(\frac{E}{C^{\wedge_{E} a}} \otimes \Delta_{b, c}^{E_{C}}\right) \Delta_{a, b+c}^{E_{C}} .
$$




$$
\left(\frac{E}{i_{C^{\wedge} E^{a}}^{C^{a}}} \otimes C_{b}\right) \circ \Delta_{a, b}^{E_{C}}=\Delta_{a+1, b}^{E_{C}} \circ \frac{E}{i_{C^{\wedge} E^{a+b}}^{C^{a} E^{a+b+1}}}, \quad\left(C_{a} \otimes \frac{E}{i_{C^{\wedge} E^{b}}^{C^{\wedge} E^{b+1}}}\right) \circ \Delta_{a, b}^{E_{C}}=\Delta_{a, b+1}^{E_{C}} \circ \frac{E}{i_{C^{\wedge} E^{a+b}}^{C^{a} E^{a+b+1}}} .
$$

$$
\left(\frac{E}{C^{\wedge} d} \otimes \varepsilon\right) \Delta_{d, 0}^{E_{C}}=r_{\frac{E}{C^{\wedge} E^{d}}}^{-1}, \quad\left(\varepsilon \otimes \frac{E}{C^{\wedge}{ }^{\wedge} d}\right) \Delta_{0, d}^{E_{C}}=l_{\frac{E}{C^{\wedge} E^{d}}}^{-1}
$$

THEOREM 2.10. Let $\mathcal{M}$ be a cocomplete coabelian monoidal category such that the tensor product commutes with direct sums.

Let $\left(C, i_{C}^{E}\right)$ be a subcoalgebra of a coalgebra $(E, \Delta, \varepsilon)$ in a coabelian monoidal category $\mathcal{M}$. For every $n \in \mathbb{N}$, we set

$$
g r_{C}^{n} E=\frac{C^{\wedge_{E} n+1}}{C^{\wedge_{E}} n} .
$$

Then $E_{C}=\oplus_{n \in \mathbb{N}} \frac{E}{C^{\wedge} E^{n}}$ is a graded coalgebra, there is a unique coalgebra structure on $\operatorname{rr}_{C} E:=$ $\oplus_{n \in \mathbb{N}} g r_{C}^{n} E$ such that

is a coalgebra homomorphism and

$$
\oplus_{n \in \mathbb{N}} \frac{i_{C^{\wedge} E^{n+1}}^{E}}{C^{\wedge} E^{n}}: g r_{C} E \rightarrow E_{C}
$$

(1) $g r_{C} E$ is a graded coalgebra such that $\oplus_{n \in \mathbb{N}} \frac{i_{C^{A_{E}}{ }^{n+1}}}{C^{\wedge} E^{n}}$ is a graded homomorphism;

$$
\left(\frac{i_{C^{\wedge}{ }^{a+1}}^{E}}{C^{\wedge_{E} a}} \otimes \frac{i_{C^{\wedge}{ }^{b+1}}^{E}}{C^{\wedge_{E} b}}\right) \circ \Delta_{a, b}^{g r_{C} E}=\Delta_{a, b}^{E_{C}} \circ \frac{i_{C^{\wedge E^{a+b+1}}}^{E}}{C^{\wedge_{E} a+b}}
$$

(3) $\varepsilon_{g r_{C} E}=\varepsilon_{E} \circ i_{C}^{E} \circ p_{0}^{g r_{C} E}$.

Moreover $g r_{C} E$ is a strongly $\mathbb{N}$-graded coalgebra.

Proof. By (13), (14) and (15), we can apply Lemma 2.4 to the family $\left(\frac{E}{C^{\wedge} E^{n}}\right)_{n \in \mathbb{N}}$. It remains to prove the last assertion.

From 2), since both $\frac{i_{C^{A}{ }^{a} a+b+1}}{C^{\wedge} E^{a+b}}$ and $\Delta_{a, b}^{E_{C}}$ are monomorphisms, we get that $\Delta_{a, b}^{g r_{C} E}$ is a monomorphism too, for every $a, b \in \mathbb{N}$. Thus $g r_{C} E$ is a strongly $\mathbb{N}$-graded coalgebra.

Definition 2.11. Let $\left(C, i_{C}^{E}\right)$ be a subcoalgebra of a coalgebra $(E, \Delta, \varepsilon)$ in a cocomplete coabelian monoidal category $\mathcal{M}$ such that the tensor product commutes with direct sums.

The strongly $\mathbb{N}$-graded coalgebra $g r_{C} E$ defined in Theorem 2.10 will be called the associated graded coalgebra (of $E$ with respect to $C$ ).

TheOREM 2.12. Let $\mathcal{M}$ be a cocomplete and complete coabelian monoidal category satisfying AB5 such that the tensor product commutes with direct sums. Let $\left(C, i_{C}^{E}\right)$ be a subcoalgebra of a coalgebra $(E, \Delta, \varepsilon)$ in $\mathcal{M}$ and let $\operatorname{gr}_{C} E$ be the associated graded coalgebra.

Let

$$
T^{c}:=T_{C}^{c}\left(\frac{C \wedge_{E} C}{C}\right)
$$

be the cotensor coalgebra. Then there is a unique coalgebra homomorphism

$$
\psi: g r_{C} E \rightarrow T_{C}^{c}\left(\frac{C \wedge_{E} C}{C}\right)
$$

such that $p_{0}^{T^{c}} \circ \psi=p_{0}^{g r_{C} E}$ and $p_{1}^{T^{c}} \circ \psi=p_{1}^{g r_{C} E}$.

Moreover $\psi$ is a graded coalgebra homomorphism with

$$
\psi_{m}=\left(p_{1}^{g r_{C} E}\right)^{\square m} \circ \bar{\Delta}_{g r_{C} E}^{m-1} \circ i_{m}^{g r_{C} E} \text { for every } m \in \mathbb{N}
$$

and the following equivalent assertions hold.

(a) $g_{C} E$ is a strongly $\mathbb{N}$-graded coalgebra.

$\left(a^{\prime}\right) \Delta_{a, 1}^{g r_{C} E}: g r_{C}^{a+1}(E) \rightarrow g r_{C}^{a}(E) \otimes g r_{C}^{1}(E)$ is a monomorphism for every $a \in \mathbb{N}$.

(b) $\psi_{n}$ is a monomorphism for every $n \in \mathbb{N}$. 
(c) $\psi$ is a monomorphism.

(d) $\oplus_{0 \leq i \leq n-1} g r_{C}^{i} E=C^{\wedge_{g r_{C} E}^{n}}$, for every $n \geq 1$.

(e) $\oplus_{0 \leq i \leq 1} g r_{C}^{i} E=C \oplus \frac{C \wedge_{E} C}{C}=C^{\wedge_{g r_{C} E}^{2}}$.

Proof. By Theorem 2.10, (a) holds. We conclude by applying [AM1, Theorem 2.22].

\section{The Associated Graded Algebra}

3.1. Let $\mathcal{M}$ be a cocomplete abelian monoidal category such that the tensor product commutes with direct sums.

Recall that a graded algebra in $\mathcal{M}$ is an algebra $(A, m, u)$ where

$$
A=\oplus_{n \in \mathbb{N}} A_{n}
$$

is a graded object of $\mathcal{M}$ such that $m: A \otimes A \rightarrow A$ is a graded homomorphism i.e. there exists a family $\left(m_{n}\right)_{n \in \mathbb{N}}$ of morphisms

$$
m_{n}^{A}=m_{n}: \oplus_{a+b=n}\left(A_{a} \otimes A_{b}\right)=(A \otimes A)_{n} \rightarrow A_{n} \text { such that } m=\oplus_{n \in \mathbb{N}} m_{n} .
$$

We set

$$
m_{a, b}^{A}:=\left(A_{a} \otimes A_{b} \stackrel{\gamma_{a, b}^{A, A}}{\rightarrow}(A \otimes A)_{a+b} \stackrel{m_{a+b}}{\rightarrow} A_{a+b}\right) .
$$

A homomorphism $f:\left(A, m_{A}, u_{A}\right) \rightarrow\left(B, m_{B}, u_{B}\right)$ of algebras is a graded algebra homomorphism if it is a graded homomorphism too.

Definition 3.2. Let $\left(A=\oplus_{n \in \mathbb{N}} A_{n}, m, u\right)$ be a graded algebra in $\mathcal{M}$. In analogy with the group graded case, we say that $A$ is a strongly $\mathbb{N}$-graded algebra whenever

$m_{i, j}^{A}: A_{i} \otimes A_{j} \rightarrow A_{i+j}$ is an epimorphism for every $i, j \in \mathbb{N}$,

where $m_{i, j}^{A}$ is the morphism of Definition 3.1.

Proposition 3.3. [AM1, Proposition 3.4] Let $\mathcal{M}$ be a cocomplete abelian monoidal category such that the tensor product commutes with direct sums.

1) Let $A=\oplus_{n \in \mathbb{N}} A_{n}$ be a graded object of $\mathcal{M}$ such that there exists a family $\left(m_{a, b}\right)_{a, b \in \mathbb{N}}$

$$
m_{a, b}^{A}: A_{a} \otimes A_{b} \rightarrow A_{a+b},
$$

of morphisms and a morphism $u_{0}^{A}: \mathbf{1} \rightarrow A_{0}$ which satisfy

$$
\begin{aligned}
& m_{a+b, c}^{A} \circ\left(m_{a, b}^{A} \otimes A_{c}\right)=m_{a, b+c}^{A} \circ\left(A_{a} \otimes m_{b, c}^{A}\right), \\
& m_{d, 0}^{A} \circ\left(A_{d} \otimes u_{0}^{A}\right)=r_{A_{d}}, \quad m_{0, d}^{A} \circ\left(u_{0}^{A} \otimes A_{d}\right)=l_{A_{d}},
\end{aligned}
$$

for every $a, b, c \in \mathbb{N}$. Then there exists a unique morphism $m_{A}: A \otimes A \rightarrow A$ such that

$$
m_{A} \circ\left(i_{a}^{A} \otimes i_{b}^{A}\right)=i_{a+b}^{A} \circ m_{a, b}^{A}, \text { for every } a, b \in \mathbb{N}
$$

holds.

Moreover $\left(A=\oplus_{n \in \mathbb{N}} A_{n}, m_{A}, u_{A}=i_{0}^{A} \circ u_{0}^{A}\right)$ is a graded algebra.

2) If $A$ is a graded algebra then

$$
p_{a+b}^{A} \circ m_{A}=\sum_{a+b=n} m_{a, b}^{A} \circ\left(p_{a}^{A} \otimes p_{b}^{A}\right)
$$

holds, $u_{A}=i_{0}^{A} p_{0}^{A} u_{A}$ so that $u_{A}$ is a graded homomorphism, and we have that (17) and (18) hold for every $a, b, c \in \mathbb{N}$, where $u_{0}^{A}=p_{0}^{A} u_{A}$.

Moreover $\left(A_{0}, m_{0}=m_{0,0}^{A}, u_{0}^{A}=p_{0}^{A} u_{A}\right)$ is an algebra in $\mathcal{M}, p_{0}^{A}$ is an algebra homomorphism and, for every $n \in \mathbb{N},\left(A_{n}, m_{0, n}^{A}, m_{n, 0}^{A}\right)$ is an $A_{0}$-bimodule such that $i_{n}^{A}: A_{n} \rightarrow A$ is a morphism of $A_{0}$-bimodules ( $A$ is an $A_{0}$-bimodule through $i_{0}^{A}$ ). 
LEMMA 3.4. Let $\mathcal{M}$ be a cocomplete abelian monoidal category such that the tensor product commutes with direct sums. Let $\left(\left(A_{a}\right)_{a \in \mathbb{N}},\left(a_{A_{a}}^{A_{b}}\right)_{a, b \in \mathbb{N}}\right)$ be a direct system in $\mathcal{M}$ where, for $a \leq b$, $i_{A_{a}}^{A_{b}}: A_{a} \rightarrow A_{b}$ is a monomorphism. Assume that there exists a family $\left(m_{a, b}^{A}\right)_{a, b \in \mathbb{N}}$

$$
m_{a, b}^{A}: A_{a} \otimes A_{b} \rightarrow A_{a+b},
$$

of morphisms and a morphism $u_{0}^{A}: \mathbf{1} \rightarrow A_{0}$ which satisfy (17), (18),

(21) $m_{a+1, b}^{A} \circ\left(i_{A_{a}}^{A_{a+1}} \otimes A_{b}\right)=i_{A_{a+b}}^{A_{a+b+1}} \circ m_{a, b}^{A} \quad$ and $\quad m_{a, b+1}^{A} \circ\left(A_{a} \otimes i_{A_{b}}^{A_{b+1}}\right)=i_{A_{a+b}}^{A_{a+b+1}} \circ m_{a, b}^{A}$ for every $a, b, c \in \mathbb{N}$. Set $A_{-1}:=0$.

Then $A=\oplus_{n \in \mathbb{N}} A_{n}$ is a graded algebra and there are unique algebra structure on $\oplus_{n \in \mathbb{N}} \frac{A_{n}}{A_{n-1}}$ such that

$$
\oplus_{n \in \mathbb{N}} p_{A_{n-1}}^{A_{n}}: \oplus_{n \in \mathbb{N}} A_{n} \rightarrow \oplus_{n \in \mathbb{N}} \frac{A_{n}}{A_{n-1}}
$$

is an algebra homomorphism. Moreover

1) $E=\oplus_{n \in \mathbb{N}} \frac{A_{n}}{A_{n-1}}$ is a graded algebra such that $\oplus_{n \in \mathbb{N}} p_{A_{n-1}}^{A_{n}}$ is a graded homomorphism;

2) $m_{a, b}^{E} \circ\left(p_{A_{a-1}}^{A_{a}} \otimes p_{A_{b-1}}^{A_{b}}\right)=p_{A_{a+b-1}}^{A_{a+b}} \circ m_{a, b}^{A}$;

3) $u_{E}=i_{0}^{E} \circ p_{A_{-1}}^{A_{0}} \circ u_{0}^{A}$.

Proof. It is analogous to that of Lemma 2.4.

LEMMA 3.5. Let $\mathcal{M}$ be a cocomplete abelian monoidal category such that the tensor product commutes with direct sums. Let $\left(\left(A_{a}\right)_{a \in \mathbb{N}},\left(i_{A_{a}}^{A_{b}}\right)_{a, b \in \mathbb{N}}\right)$ be an inverse system in $\mathcal{M}$ where, for $a \leq b$, $i_{A_{b}}^{A_{a}}: A_{b} \rightarrow A_{a}$ is a monomorphism. Assume that there exists a family $\left(m_{a, b}^{A}\right)_{a, b \in \mathbb{N}}$

$$
m_{a, b}^{A}: A_{a} \otimes A_{b} \rightarrow A_{a+b},
$$

of morphisms and a morphism $u_{0}^{A}: \mathbf{1} \rightarrow A_{0}$ which satisfy (17), (18),

(22) $m_{a, b}^{A} \circ\left(i_{A_{a+1}}^{A_{a}} \otimes A_{b}\right)=i_{A_{a+b+1}}^{A_{a+b}} \circ m_{a+1, b}^{A} \quad$ and $\quad m_{a, b}^{A} \circ\left(A_{a} \otimes i_{A_{b+1}}^{A_{b}}\right)=i_{A_{a+b+1}}^{A_{a+b}} \circ m_{a, b+1}^{A}$ for every $a, b, c \in \mathbb{N}$. Set $A_{-1}:=0$.

Then $A=\oplus_{n \in \mathbb{N}} A_{n}$ is a graded algebra and there are unique algebra structure on $\oplus_{n \in \mathbb{N}} \frac{A_{n}}{A_{n+1}}$ such that

is an algebra homomorphism. Moreover

$$
\oplus_{n \in \mathbb{N}} p_{A_{n+1}}^{A_{n}}: \oplus_{n \in \mathbb{N}} A_{n} \rightarrow \oplus_{n \in \mathbb{N}} \frac{A_{n}}{A_{n+1}}
$$

1) $E=\oplus_{n \in \mathbb{N}} \frac{A_{n}}{A_{n+1}}$ is a graded algebra such that $\oplus_{n \in \mathbb{N}} p_{A_{n+1}}^{A_{n}}$ is a graded homomorphism;

2) $m_{a, b}^{E} \circ\left(p_{A_{a+1}}^{A_{a}} \otimes p_{A_{b+1}}^{A_{b}}\right)=p_{A_{a+b+1}}^{A_{a+b}} \circ m_{a, b}^{A}$;

3) $u_{E}=i_{0}^{E} \circ p_{A_{1}}^{A_{0}} \circ u_{0}^{A}$.

Proof. It is similar to that of Lemma 3.4.

THEOREM 3.6. With hypothesis and notations of Lemma 3.4, the following assertions are equivalent.

(1) $A=\oplus_{n \in \mathbb{N}} A_{n}$ is a strongly $\mathbb{N}$-graded algebra.

(2) $E=\oplus_{n \in \mathbb{N}} \frac{A_{n}}{A_{n-1}}$ is a strongly $\mathbb{N}$-graded algebra.

Proof. It is analogous to that of Theorem 2.7.

3.7. Recall from AMS2 that an ideal of an algebra $(A, m, u)$ in a monoidal category $(\mathcal{M}, \otimes, \mathbf{1})$ is a pair $\left(I, i_{I}^{A}\right)$ where $I$ is an $A$-bimodule and

$$
i_{I}^{A}: I \rightarrow A
$$


is a morphism of $A$-bimodules which is a monomorphism in $\mathcal{M}$.

A morphism $f: I \rightarrow J$ in ${ }_{A} \mathcal{M}_{A}$, where $I, J$ are two ideals, is called a morphism of ideals whenever

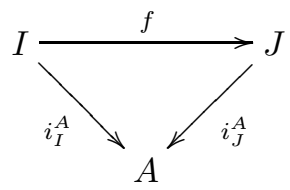

Note that $f$ is a monomorphism in $\mathcal{M}$ as $i_{I}^{A}$ is a monomorphism. Moreover $f$ is unique, as $i_{J}^{A}$ is a monomorphism.

3.8. Let $\mathcal{M}$ be an abelian monoidal category.

Let $\left(I, i_{I}^{A}\right)$ and $\left(J, i_{J}^{A}\right)$ be two subobjects of an algebra $(A, m, u)$. Set

$$
\begin{gathered}
m_{I, J}:=m\left(i_{I}^{A} \otimes i_{J}^{A}\right): I \otimes J \rightarrow A \\
\left(Q_{I, J}, \pi_{I, J}\right)=\operatorname{Coker}\left(m_{I, J}\right), \quad \pi_{I, J}^{A}: A \rightarrow Q_{I, J} \\
\left(I J, i_{I J}^{A}\right)=\operatorname{Ker}\left(\pi_{I, J}^{A}\right)=\operatorname{Im}\left(m_{I, J}\right), \quad i_{I J}^{A}: I J \rightarrow A
\end{gathered}
$$

The subobject $\left(I J, i_{I J}^{A}\right)$ of $A$ is called the product of $I$ and $J$.

Moreover, we have the following exact sequence:

$$
0 \longrightarrow I J \stackrel{i_{I J}^{A}}{\longrightarrow} A \stackrel{\pi_{I, J}^{A}}{\longrightarrow} Q_{I, J} \longrightarrow 0 .
$$

Since $\left(I J, i_{I J}^{A}\right)=\operatorname{Ker}\left(\pi_{I, J}^{A}\right)$ and $\pi_{I, J}^{A} m_{I, J}=0$, by the universal property of the kernel, there is a unique morphism $\bar{m}_{I, J}: I \otimes J \rightarrow I J$ such that the following diagram

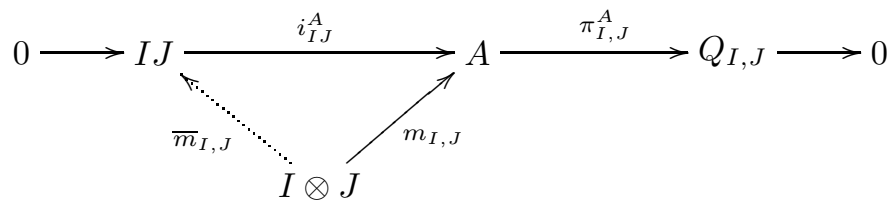

is commutative. Since $I J=\operatorname{Im}\left(m_{I, J}\right)$, it comes out that $\bar{m}_{I, J}$ is an epimorphism.

Consider the case $I=A$.

Assume now that $\left(I, i_{I}^{A}\right)$ and $\left(J, i_{J}^{A}\right)$ are two ideals of $(A, m, u)$.

In this case $\left(I J, i_{I J}^{A}\right)$ is an ideal of $A$ and $m_{I, J} \in{ }_{A} \mathcal{M}_{A}$ so that it is straightforward to prove that $Q_{I, J}$ is an algebra and that $\pi_{I, J}$ and algebra homomorphism.

Since $i_{J}^{A}$ is a morphism in ${ }_{A} \mathcal{M}$, we have

$$
m_{A, J}=m \circ\left(\operatorname{Id}_{A} \otimes i_{J}^{A}\right)=i_{J}^{A} \circ \mu_{J}^{l} .
$$

Since $i_{J}^{A}$ is a monomorphism and $\mu_{J}^{l}$ an epimorphism, we deduce that

$$
\left(A J, i v_{A J}\right)=\operatorname{Im}\left(m_{A, J}\right)=\left(J, i_{J}^{A}\right) .
$$

Analogously, in the case $J=A$, one has

$$
\left(I A, i_{I A}^{A}\right)=\left(I, i_{I}^{A}\right) .
$$

3.9. Let $A$ be an algebra in an abelian monoidal category $\mathcal{M}$ and let $\left(I, i_{I}^{A}\right)$ be a subobject of $A$. We can define (see [AMS2]) the $n$-th power $\left(I^{n}, i_{I^{n}}^{A}\right)$ of $I$, where $i_{I^{n}}^{A}: I^{n} \rightarrow A$. By Definition

$$
I^{0}=A \quad \text { and } \quad I^{n}=I^{n-1} I, \text { for every } n \geq 1 .
$$

For every subobject $I, J, K$ of $A$ one can check that $\left((I J) K, i_{(I J) K}^{A}\right)$ and $\left(I(J K), i_{I(J K)}^{A}\right)$ are isomorphic and thus can be identified. Therefore $I^{i} I^{j}=I^{i+j}$ and we can consider $\bar{m}_{I^{i}, I^{j}}: I^{i} \otimes I^{j} \rightarrow$ $I^{i+j}$. We set

$$
m_{i, j}^{I}:=\bar{m}_{I^{i}, I^{j}}: I^{i} \otimes I^{j} \rightarrow I^{i+j}
$$


Assume now that $\left(I, i_{I}^{A}\right)$ is an ideal of $A$. Then there is a (unique) morphism of ideals

$$
i_{I^{n+1}}^{I^{n}}: I^{n+1} \rightarrow I^{n} \text {, for every } n \in \mathbb{N} .
$$

Since $m$ is associative and by definition of $m_{i, j}^{I}$, it is straightforward to prove that $m_{i, j}^{I}$ fulfills

$$
\begin{aligned}
& m_{a+b, c}^{I}\left(m_{a, b}^{I} \otimes I^{c}\right)=m_{a, b+c}^{I}\left(I^{a} \otimes m_{b, c}^{I}\right) . \\
& m_{d, 0}^{I}\left(I^{d} \otimes u\right)=r_{I^{d}}, \quad m_{0, d}^{I}\left(u \otimes I^{d}\right)=l_{I^{d}} \\
& m_{a, b}^{I} \circ\left(i_{I^{a+1}}^{I^{a}} \otimes I^{b}\right)=i_{I^{a+b+1}}^{I^{a+b}} \circ m_{a+1, b}^{I}, \quad m_{a, b}^{I} \circ\left(I^{a} \otimes i_{I^{b+1}}^{I^{b}}\right)=i_{I^{a+b+1}}^{I^{a+b}} \circ m_{a, b+1}^{I}
\end{aligned}
$$

TheOREM 3.10. Let $\mathcal{M}$ be a cocomplete abelian monoidal category such that the tensor product commutes with direct sums.

Let $(A, m, u)$ be an algebra in $\mathcal{M}$ and let $\left(I, i_{I}\right)$ be an ideal of $A$ in $\mathcal{M}$. For every $n \in \mathbb{N}$, we set

$$
g r_{I}^{n} A=\frac{I^{n}}{I^{n+1}} .
$$

Then $\oplus_{n \in \mathbb{N}} I^{n}$ is a graded algebra and there are unique algebra structure on $g r_{I} A:=\oplus_{n \in \mathbb{N}} g r_{I}^{n} A$ such that

is an algebra homomorphism. Moreover

$$
\oplus_{n \in \mathbb{N}} p_{I^{n+1}}^{I^{n}}: \oplus_{n \in \mathbb{N}} I^{n} \rightarrow g r_{I} A
$$

1) $\operatorname{gr}_{I} A$ is a graded algebra such that $\oplus_{n \in \mathbb{N}} \pi_{n}^{I}$ is a graded homomorphism;

2)

$$
m_{a, b}^{g r_{I} A} \circ\left(p_{I^{a+1}}^{I^{a}} \otimes p_{I^{b+1}}^{I^{b}}\right)=p_{I^{a+b+1}}^{I^{a+b}} \circ m_{a, b}^{I}, \text { for every } a, b \in \mathbb{N}
$$

3) $u_{g r_{I} A}=i_{0}^{g r_{I} A} \circ p_{I}^{A} \circ u$

Moreover $\operatorname{gr}_{I} A$ is a strongly $\mathbb{N}$-graded algebra.

Proof. By (24), (25) and (26), we can apply Lemma 3.5 to the family $\left(I^{n}\right)_{n \in \mathbb{N}}$. It remains to prove the last assertion. From 2), since both $p_{I^{a+b+1}}^{I^{a+b}}$ and $m_{a, b}^{I}$ are epimorphisms, we get that $m_{a, b}^{g r_{I} A}$ is an epimorphism too, for every $a, b \in \mathbb{N}$. Thus $\operatorname{gr}_{I} A$ is a strongly $\mathbb{N}$-graded algebra.

Definition 3.11. Let $\mathcal{M}$ be a cocomplete abelian monoidal category such that the tensor product commutes with direct sums.

Given an ideal $I$ of an algebra $A$ in $\mathcal{M}$, the strongly $\mathbb{N}$-graded algebra $\operatorname{gr}_{I} A$ defined in Theorem 3.10 will be called the associated graded algebra (of $A$ with respect to $I$ ).

THEOREM 3.12. Let $\mathcal{M}$ be a cocomplete abelian monoidal category such that the tensor product commutes with direct sums. Let I be an ideal of an algebra $A$ in $\mathcal{M}$ an let $\operatorname{gr}_{I} A$ be the associated graded algebra.

Let

$$
T:=T_{\frac{A}{I}}\left(\frac{I}{I^{2}}\right)
$$

be the tensor algebra. Then there is a unique algebra homomorphism

$$
\varphi: T_{\frac{A}{T}}\left(\frac{I}{I^{2}}\right) \rightarrow g r_{I} A
$$

such that $\varphi \circ i_{0}^{T}=i_{0}^{g r_{I} A}$ and $\varphi \circ i_{1}^{T}=i_{1}^{g r_{I} A}$.

Moreover $\varphi$ is a graded algebra homomorphism with

$$
\varphi_{t}=p_{t}^{g r_{I} A} \circ \bar{m}_{g r_{I} A}^{t-1} \circ\left(i_{1}^{g r_{I} A}\right)^{\otimes_{A_{0}} t} \text { for every } t \in \mathbb{N}
$$

and the following equivalent assertions hold.

(a) $\operatorname{gr}_{I} A$ is a strongly $\mathbb{N}$-graded algebra.

(a) $m_{a, 1}^{g r_{I} A}: g r_{I}^{a} A \otimes g r_{I}^{1} A \rightarrow g r_{I}^{a+1} A$ is an epimorphism for every $a \in \mathbb{N}$.

(b) $\varphi_{n}$ is an epimorphism for every $n \in \mathbb{N}$.

(c) $\varphi$ is an epimorphism.

(d) $\oplus_{i \geq n} g r_{I}^{i} A=\left(\oplus_{i \geq 1} g r_{I}^{i} A\right)^{n}$, for every $n \in \mathbb{N}$. 
(e) $\oplus_{i \geq 2} g r_{I}^{i} A=\left(\oplus_{i \geq 1} g r_{I}^{i} A\right)^{2}$.

Proof. By Theorem 3.10, (a) holds. We conclude by applying [AM1, Theorem 3.11].

\section{The Associated Graded Coalgebra of a Bialgebra With Respect to a SubBialgEBRA}

Lemma 4.1. Let $(\mathcal{M}, c)$ be a cocomplete abelian braided monoidal category such that the tensor product commutes with direct sums. Consider in $(\mathcal{M}, c)$ a datum $\left(B, m_{B}, u_{B}, \Delta_{B}, \varepsilon_{B}\right)$ consisting of a graded object $B$ with graduation defined by $\left(B_{k}\right)_{k \in \mathbb{N}}$ such that, with respect to this graduation,

- $\left(B, m_{B}, u_{B}\right)$ is a graded algebra and

- $\left(B, \Delta_{B}, \varepsilon_{B}\right)$ is a graded coalgebra.

Assume that for every $a, b \in \mathbb{N}$,

$$
\begin{aligned}
& \sum_{s+t=a+b}\left(i_{s} \otimes i_{t}\right) \Delta_{s, t} m_{a, b} \\
&= \sum_{s^{\prime}+t^{\prime}=a} \sum_{s^{\prime \prime}+t^{\prime \prime}=b}\left(i_{s^{\prime}+s^{\prime \prime}} \otimes i_{t^{\prime}+t^{\prime \prime}}\right) \circ\left(m_{s^{\prime}, s^{\prime \prime}} \otimes m_{t^{\prime}, t^{\prime \prime}}\right) \circ\left(B_{s^{\prime}} \otimes c_{B_{t^{\prime}}, B_{s^{\prime \prime}}} \otimes B_{t^{\prime \prime}}\right) \circ\left(\Delta_{s^{\prime}, t^{\prime}} \otimes \Delta_{s^{\prime \prime}, t^{\prime \prime}}\right) \\
& \varepsilon_{0} m_{0,0}=m_{\mathbf{1}}\left(\varepsilon_{0} \otimes \varepsilon_{0}\right) .
\end{aligned}
$$

Then $B$ is a graded braided bialgebra in $(\mathcal{M}, c)$.

Proof. It follows easily by using (19), (6).

TheOREM 4.2. Let $\mathcal{M}$ be an coabelian monoidal category. Let $\left(\left(X_{i}\right)_{i \in \mathbb{N}},\left(\xi_{i}^{j}\right)_{i, j \in \mathbb{N}}\right)$ be a direct system in $\mathcal{M}$ where, for $i \leq j, \xi_{i}^{j}: X_{i} \rightarrow X_{j}$.

Let $\left(\xi_{i}: X_{i} \rightarrow X\right)_{i \in \mathbb{N}}$ be a compatible family of morphisms with respect to the given direct system. Assume that

- $\xi_{i}^{j}: X_{i} \rightarrow X_{j}$ is a split monomorphism for every $i \leq j$,

- $X_{0}=0$,

- $\xi_{i}: X_{i} \rightarrow X$ is a monomorphism for every $i \in \mathbb{N}$

and denote by $\tau_{i}: X \rightarrow \frac{X}{X_{i}}$ the canonical projection for every $i \in \mathbb{N}$.

Then, for every $n \in \mathbb{N}$, the following sequence is exact.

$$
\bigoplus_{a+b=n+1} X_{a} \otimes X_{b} \stackrel{\nabla\left[\left(\xi_{a} \otimes \xi_{b}\right)_{a+b=n+1}\right]}{\longrightarrow} X \otimes X \stackrel{\Delta\left[\left(\tau_{a} \otimes \tau_{b}\right)_{a+b=n}\right]}{\longrightarrow} \bigoplus_{a+b=n} \frac{X}{X_{a}} \otimes \frac{X}{X_{b}} .
$$

Proof. Apply [AM3, Theorem 3.1].

NotATIONs 4.3. In this section, from now on, the following assumptions and notations will be used.

$(\mathcal{M}, c)$ is a cocomplete abelian coabelian braided monoidal category such that the tensor product commutes with direct sums.

Let $i_{B}^{E}: B \hookrightarrow E$ be a monomorphism in $\mathcal{M}$ which is a braided bialgebra homomorphism in $\mathcal{M}$ and let $i_{B^{\wedge_{E}}}^{E}: B^{\wedge_{E} n} \rightarrow E$ and $i_{B^{\wedge} E^{a}}^{{\wedge^{b}}^{b}}: B^{\wedge_{E} a} \rightarrow B^{\wedge_{E} b}(a \leq b)$ be the canonical injections.

Assume that $i_{B^{\wedge} E^{a}}^{\wedge^{A^{b}}}$ is a split monomorphism in $\mathcal{M}$ for every $a \leq b$.

By Theorem 4.9, we have the following exact sequence

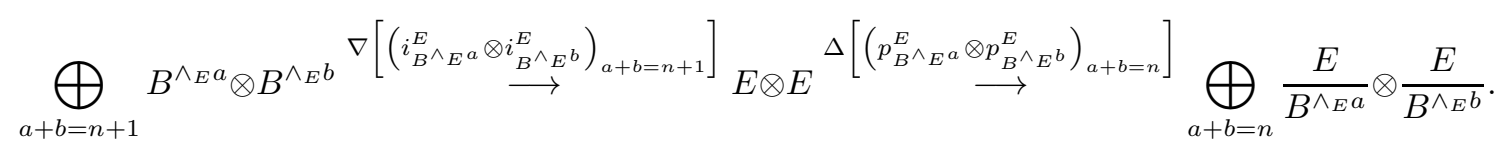

Let

$$
\left(\sum_{a+b=n+1} B^{\wedge_{E} a} \otimes B^{\wedge_{E} b}, \beta_{n}\right)=\operatorname{Im}\left\{\nabla\left[\left(i_{B^{\wedge_{E}}}^{E} \otimes i_{B^{\wedge^{b}}}^{E}\right)_{a+b=n+1}\right]\right\}
$$


and let

$$
\gamma_{n}: \bigoplus_{a+b=n+1} B^{\wedge_{E} a} \otimes B^{\wedge_{E} b} \rightarrow \sum_{a+b=n+1} B^{\wedge_{E} a} \otimes B^{\wedge_{E} b}
$$

be the unique morphism such that

$$
\beta_{n} \circ \gamma_{n}=\nabla\left[\left(i_{B^{\wedge} E^{a}}^{E} \otimes i_{B^{\wedge} E^{b}}^{E}\right)_{a+b=n+1}\right] .
$$

Since $\left(B^{\wedge_{E} a+b}, i_{B^{\wedge E^{a+b}}}^{E}\right)=\operatorname{ker}\left(\left(p_{B^{\wedge E^{a}}}^{E} \otimes p_{B^{\wedge E^{b}}}^{E}\right) \Delta_{E}\right)$, we have $\left(p_{B^{\wedge E^{a}}}^{E} \otimes p_{B^{{ }^{E^{b}}}}^{E}\right) \circ \Delta_{E} \circ i_{B^{\wedge} E^{a+b}}^{E}=$ 0 so that

$$
\Delta\left[\left(p_{B^{\wedge} E^{a}}^{E} \otimes p_{B^{\wedge} E^{b}}^{E}\right)_{a+b=n}\right] \circ \Delta_{E} \circ i_{B^{\wedge} E^{a+b}}^{E}=0
$$

and hence, by the exactness of the sequence above, there exists a unique morphism

$$
\alpha_{n}: B^{\wedge_{E} n} \rightarrow \sum_{a+b=n+1} B^{\wedge_{E} a} \otimes B^{\wedge_{E} b}
$$

such that

$$
\Delta_{E} \circ i_{B^{\wedge} E^{n}}^{E}=\beta_{n} \circ \alpha_{n}, \text { for every } n \in \mathbb{N} .
$$

Lemma 4.4. 0) For every $s, t, u, v \in \mathbb{N}$, we have

$$
\begin{aligned}
& \left(p_{B^{\wedge} E^{s}}^{E} \otimes p_{B^{\wedge} E^{t}}^{E}\right) \circ\left(m_{E} \otimes m_{E}\right) \circ(E \otimes c \otimes E) \circ\left(\beta_{u} \otimes \beta_{v}\right) \circ\left(\gamma_{u} \otimes \gamma_{v}\right) \\
& =\nabla\left[\left(\begin{array}{c}
\left(\left[p_{B^{\wedge} E^{s}}^{E} m_{E}\left(i_{B^{\wedge} E^{a}}^{E} \otimes i_{B^{\wedge} E^{b}}^{E}\right)\right] \otimes\left[p_{B^{\wedge} E^{t}}^{E} m_{E}\left(i_{B^{\wedge} E^{c}}^{E} \otimes i_{B^{\wedge^{d}}}^{E}\right)\right]\right) \\
\circ\left(B^{\wedge^{\prime} a} \otimes c_{B^{\wedge} E^{c}, B^{\wedge} E^{b}}^{E} \otimes B^{\wedge_{E} d}\right)
\end{array}\right)_{\begin{array}{c}
a+c=u+1 \\
b+d=v+1
\end{array}}\right]
\end{aligned}
$$

1) The following relations hold.

$$
p_{B^{\wedge} E^{u+v-1}}^{E} m_{E}\left(i_{B^{\wedge} E^{u}}^{E} \otimes i_{B^{\wedge} E^{v}}^{E}\right)=0 \text { for every } u, v \in \mathbb{N}, u+v \geq 1 .
$$

2) For every $a, b \in \mathbb{N}$, there exists a unique morphism $m_{\wedge}^{a, b}: B^{\wedge_{E} a+1} \otimes B^{\wedge_{E} b+1} \rightarrow B^{\wedge_{E} a+b+1}$ such that

$$
i_{B^{\wedge} E^{a+b+1}}^{E} \circ m_{\wedge}^{a, b}=m_{E}\left(i_{B^{\wedge} E^{a+1}}^{E} \otimes i_{B^{\wedge} E^{b+1}}^{E}\right), \text { for every } a, b \in \mathbb{N} .
$$

3) For every $a, b, c, d \in \mathbb{N}$, we have

$$
\begin{aligned}
& m_{\wedge}^{a+b, c} \circ\left(m_{\wedge}^{a, b} \otimes B^{\wedge_{E} c+1}\right)=m_{\wedge}^{a, b+c} \circ\left(B^{\wedge_{E} a+1} \otimes m_{\wedge}^{b, c}\right), \\
& m_{\wedge}^{d, 0} \circ\left(B^{\wedge_{E} d+1} \otimes u_{B}\right)=r_{B^{\wedge} E^{d+1}}, \quad m_{\wedge}^{0, d} \circ\left(u_{B} \otimes B^{\wedge_{E} d+1}\right)=l_{B^{\wedge} E^{d+1}}, \\
& m_{\wedge}^{a+1, b} \circ\left(i_{B^{\wedge} E^{a+1}}^{{\wedge^{a}}^{a+2}} \otimes B^{\wedge_{E} b+1}\right)=i_{B^{\wedge} E^{a+b+1}}^{{\wedge^{a}}^{a+b+2}} \circ m_{\wedge}^{a, b}, \quad m_{\wedge}^{a, b+1} \circ\left(B^{\wedge_{E} a+1} \otimes i_{B^{\wedge} E^{b+1}}^{{\wedge^{b}}^{b+2}}\right)=i_{B^{\wedge} E^{a+b+1}}^{{\wedge}^{a+b+2}} \circ m_{\wedge}^{a, b}
\end{aligned}
$$

Proof. See the Appendix.

Proposition 4.5. $\oplus_{n \in \mathbb{N}} B^{\wedge_{E}}{ }^{n+1}$ is a graded algebra and there are unique algebra structure on $g r_{B} E=\oplus_{n \in \mathbb{N}} g r_{B}^{n} E$ such that

$$
\oplus_{n \in \mathbb{N}} p_{B^{\wedge} E^{n}}^{\wedge^{\wedge^{n+1}}}: \oplus_{n \in \mathbb{N}} B^{\wedge_{E} n+1} \rightarrow g r_{B} E
$$

is an algebra homomorphism. Moreover

1) $g r_{B} E=\oplus_{n \in \mathbb{N}} g r_{B}^{n} E$ is a graded algebra such that $\oplus_{n \in \mathbb{N}} p_{B^{\wedge} E^{n}}^{{\wedge^{n}}^{n+1}}$ is a graded homomorphism;

$$
p_{B^{\wedge} E^{a+b}}^{{B^{a}}^{a+b+1}} \circ m_{\wedge}^{a, b}=m_{a, b}^{g r_{B} E} \circ\left(p_{B^{\wedge} E^{a}}^{{\lambda_{E}}^{a+1}} \otimes p_{B^{\wedge} E^{b}}^{{\lambda^{\wedge} E^{b+1}}^{b}}\right) .
$$

3) $u_{g r_{B} E}=i_{0}^{g r_{B} E} \circ p_{B^{\wedge} E^{0}}^{B} \circ u_{B}$

Proof. By Lemma 4.4, we can apply Lemma 3.4 to the family $\left(B^{\wedge_{E} n+1}\right)_{n \in \mathbb{N}}$. 
THEOREM 4.6. Let $(\mathcal{M}, c)$ be a cocomplete and complete abelian coabelian braided monoidal category satisfying AB5. Assume that the tensor product commutes with direct sums. Let $i_{B}^{E}: B \hookrightarrow E$ be a monomorphism in $\mathcal{M}$ which is a braided bialgebra homomorphism in $\mathcal{M}$ and let $i_{B^{\wedge} E^{n}}^{{ }^{n}}: B^{\wedge_{E} n} \rightarrow E$ and $i_{B^{\wedge} E^{a}}^{{\wedge^{b}}^{b}}: B^{\wedge_{E} a} \rightarrow B^{\wedge_{E} b}(a \leq b)$ be the canonical injections. Assume that $i_{B^{\wedge} E^{a}}^{\wedge^{b}}$ is a split monomorphism in $\mathcal{M}$ for every $a \leq b$.

Then $\mathrm{gr}_{B} E$ is a graded braided bialgebra in $(\mathcal{M}, c)$.

Proof. Set $i_{n}:=i_{B^{\wedge} E^{a}}^{E}, p_{n}:=p_{B^{\wedge} E^{n}}^{E}$ and $p_{n}^{n+1}:=p_{B^{\wedge} E^{n}}^{{\wedge^{n}}^{n+1}}$.

By Theorem 2.10, $\left(g r_{B} E, \Delta_{g r_{B} E}, \varepsilon_{g r_{B} E}=\varepsilon_{C} \circ \sigma_{C}^{0} \circ p_{0}^{g r_{C} E}\right)$ is a strongly $\mathbb{N}$-graded coalgebra.

By Proposition 4.5, $\left(g r_{B} E, m_{g r_{B} E}, u_{g r_{B} E}=i_{0}^{g r_{B} E} \circ p_{0}^{1} \circ u_{B}\right)$ is a graded algebra with the same graduation defined by $\left(g r_{B}^{k} E\right)_{k \in \mathbb{N}}$.

By Lemma 4.1, in order to get that $g r_{B} E$ is a graded braided bialgebra we have to prove that

$$
\begin{aligned}
& \sum_{s+t=a+b}\left(i_{s}^{g r_{B} E} \otimes i_{t}^{g r_{B} E}\right) \circ \Delta_{s, t}^{g r_{B} E} \circ m_{a, b}^{g r_{B} E} \\
& =\sum_{s^{\prime}+t^{\prime}=a} \sum_{s^{\prime \prime}+t^{\prime \prime}=b}\left[\begin{array}{c}
\left(i_{s^{\prime}+s^{\prime \prime}}^{g r_{B} E} \otimes i_{t^{\prime}+t^{\prime \prime}}^{g r_{B} E}\right) \circ\left(m_{s^{\prime}, s^{\prime \prime}}^{g r_{B} E} \otimes m_{t^{\prime}, t^{\prime \prime}}^{g r_{B} E}\right) \circ \\
\circ\left(\frac{B^{\wedge} E^{s^{\prime}+1}}{B^{\wedge} E^{s^{\prime}}} \otimes c_{\frac{B^{\wedge} E^{t^{\prime}+1}}{B^{\wedge} E^{t^{\prime}}}, \frac{B^{\wedge} E^{s^{\prime \prime}+1}}{B^{\wedge} E^{s^{\prime \prime}}}} \otimes \frac{B^{\wedge} E^{t^{\prime \prime}+1}}{B^{\wedge} E^{t^{\prime \prime}}}\right) \circ\left(\Delta_{s^{\prime}, t^{\prime}}^{g r_{B} E} \otimes \Delta_{s^{\prime \prime}, t^{\prime \prime}}^{g r_{B} E}\right)
\end{array}\right],
\end{aligned}
$$

for every $a, b \in \mathbb{N}$. Denote by

the canonical injection. Since

$$
j_{z}: \frac{E}{B^{\wedge_{E} z}} \rightarrow \oplus_{i \in \mathbb{N}} \frac{E}{B^{\wedge_{E}} i}
$$

$$
\left(\oplus_{w \in \mathbb{N}} \frac{i_{w+1}}{B^{\wedge_{E} w}}\right) \circ i_{s}^{g r_{B} E}=j_{s} \circ \frac{i_{s+1}}{B^{\wedge_{E} s}} \text { for every } s \in \mathbb{N}
$$

and $\oplus_{w \in \mathbb{N}} \frac{i_{w+1}}{B^{\wedge} E^{w}}$ is a monomorphism (our category is an $A B 4$ category [Po, page 53]) and $p_{a}^{a+1} \otimes p_{b}^{b+1}$ is an epimorphism, the equality we have to prove is equivalent to

$$
\begin{aligned}
& \sum_{s+t=a+b}\left(j_{s} \otimes j_{t}\right) \circ\left(\frac{i_{s+1}}{B^{\wedge_{E}} s} \otimes \frac{i_{t+1}}{B^{\wedge_{E} t}}\right) \circ \Delta_{s, t}^{g r_{B} E} \circ m_{a, b}^{g r_{B} E} \circ\left(p_{a}^{a+1} \otimes p_{b}^{b+1}\right) \\
& =\sum_{s^{\prime}+t^{\prime}=a} \sum_{s^{\prime \prime}+t^{\prime \prime}=b}\left[\begin{array}{c}
\left(j_{s^{\prime}+s^{\prime \prime}} \otimes j_{t^{\prime}+t^{\prime \prime}}\right) \circ\left(\frac{i_{s^{\prime}+s^{\prime \prime}+1}}{B^{\wedge} E^{s^{\prime}+s^{\prime \prime}}} \otimes \frac{i_{t^{\prime}+t^{\prime \prime}+1}}{B^{\wedge} E^{t^{\prime}+t^{\prime \prime}}}\right) \circ\left(m_{s^{\prime}, s^{\prime \prime}}^{g r_{B} E} \otimes m_{t^{\prime}, t^{\prime \prime}}^{g r_{B} E}\right) \circ \\
\circ\left(\frac{B^{\wedge} s^{s^{\prime}+1}}{B^{\wedge} E^{s^{\prime}}} \otimes c_{\frac{B^{\wedge} E^{\prime}+1}{B^{\wedge} E^{t^{\prime}}}, \frac{B^{\wedge} E^{s^{\prime \prime}+1}}{B^{\wedge} E^{s^{\prime \prime}}}} \otimes \frac{B^{\wedge} E^{t^{\prime \prime}+1}}{B^{\wedge} E^{t^{\prime \prime}}}\right) \\
\circ\left(\Delta_{s^{\prime}, t^{\prime}}^{g r_{B} E} \otimes \Delta_{s^{\prime \prime}, t^{\prime \prime}}^{g r_{B} E}\right) \circ\left(p_{a}^{a+1} \otimes p_{b}^{b+1}\right)
\end{array}\right]
\end{aligned}
$$

By using (33), (16), (32), the compatibility between $\Delta_{E}$ and $m_{E}$, and (29), the first term rewrites as

$$
\sum_{s+t=a+b}\left(j_{s} \otimes j_{t}\right) \circ\left(p_{s} \otimes p_{t}\right) \circ\left(m_{E} \otimes m_{E}\right) \circ\left(E \otimes c_{E, E} \otimes E\right) \circ\left(\beta_{a+1} \otimes \beta_{b+1}\right) \circ\left(\alpha_{a+1} \otimes \alpha_{b+1}\right)
$$

On the other hand, in view of (46), the second term rewrites as $\Xi \circ\left(\alpha_{a+1} \otimes \alpha_{b+1}\right)$ where

$$
\Xi=\sum_{\substack{s^{\prime}+t^{\prime}=a \\
s^{\prime \prime}+t^{\prime \prime}=b}}\left[\begin{array}{c}
\left(j_{s^{\prime}+s^{\prime \prime}} \otimes j_{t^{\prime}+t^{\prime \prime}}\right) \circ\left(\frac{i_{s^{\prime}+s^{\prime \prime}+1}}{B^{\wedge} E^{s^{\prime}+s^{\prime \prime}}} \otimes \frac{i_{t^{\prime}+t^{\prime \prime}+1}}{B^{\wedge} E^{t^{\prime}+t^{\prime \prime}}}\right) \circ\left(m_{s^{\prime}, s^{\prime \prime}}^{g r_{B} E} \otimes m_{t^{\prime}, t^{\prime \prime}}^{g r_{B} E}\right) \circ \\
\circ\left(\frac{B^{\wedge} E^{s^{\prime}+1}}{B^{\wedge} E^{s^{\prime}}} \otimes c_{\frac{B^{\wedge} E^{t^{\prime}+1}}{B^{\wedge} E^{t^{\prime}}}, \frac{B^{\wedge} E^{s^{\prime \prime}+1}}{B^{\wedge} E^{s^{\prime \prime}}}} \otimes \frac{B^{\wedge} E^{t^{\prime \prime}+1}}{B^{\wedge} E^{t^{\prime \prime}}}\right) \circ\left(\theta_{s^{\prime}, t^{\prime}} \otimes \theta_{s^{\prime \prime}, t^{\prime \prime}+1}\right)
\end{array}\right] .
$$

We will prove that

$$
\Xi=\sum_{s+t=a+b}\left(j_{s} \otimes j_{t}\right) \circ\left(p_{s} \otimes p_{t}\right) \circ\left(m_{E} \otimes m_{E}\right) \circ\left(E \otimes c_{E, E} \otimes E\right) \circ\left(\beta_{a+1} \otimes \beta_{b+1}\right)
$$


Since $\gamma_{a+1} \otimes \gamma_{b+1}$ is an epimorphism, equivalently we will prove that

$$
\begin{aligned}
& \Xi \circ\left(\gamma_{a+1} \otimes \gamma_{b+1}\right) \\
& \sum_{s+t=a+b}\left(j_{s} \otimes j_{t}\right) \circ\left(p_{s} \otimes p_{t}\right) \circ\left(m_{E} \otimes m_{E}\right) \circ\left(E \otimes c_{E, E} \otimes E\right) \circ\left(\beta_{a+1} \otimes \beta_{b+1}\right) \circ\left(\gamma_{a+1} \otimes \gamma_{b+1}\right) .
\end{aligned}
$$

This is achieved by using (47), (33), (32), naturality of braiding and (30).

In view of Lemma 4.1, it remains to prove that

$$
\varepsilon_{0}^{g r_{B} E} \circ m_{0,0}^{g r_{B} E}=m_{\mathbf{1}} \circ\left(\varepsilon_{0}^{g r_{B} E} \otimes \varepsilon_{0}^{g r_{B} E}\right) .
$$

This follows easily once proved that $\varepsilon_{0}^{g r_{B} E}=\varepsilon_{B}$ and $m_{0,0}^{g r_{B} E}=m_{B}$.

In view of Theorem 2.10, we have

$$
\varepsilon_{0}^{g r_{B} E}=\varepsilon_{g r_{C} E} \circ i_{0}^{g r_{B} E}=\varepsilon_{E} \circ \sigma_{B}^{0} \circ p_{0}^{g r_{B} E} \circ i_{0}^{g r_{B} E}=\varepsilon_{E} \circ i_{B}^{E}=\varepsilon_{B} .
$$

Since

$$
i_{B}^{E} \circ m_{\wedge}^{0,0} m_{E}\left(i_{B}^{E} \otimes i_{B}^{E}\right)=i_{B}^{E} m_{B}
$$

we get that $m_{\wedge}^{0,0}=m_{B}$ and hence

$$
m_{0,0}^{g r_{B} E}=m_{0,0}^{g r_{B} E} \circ\left(p_{0}^{1} \otimes p_{0}^{1}\right) \stackrel{(33)}{=} p_{0}^{1} \circ m_{\wedge}^{0,0}=m_{\wedge}^{0,0}=m_{B} .
$$

THEOREM 4.7. Let $(\mathcal{M}, c)$ be a cocomplete and complete abelian coabelian braided monoidal category satisfying AB5. Assume that the tensor product commutes with direct sums. Let $i_{B}^{E}: B \hookrightarrow E$ be a monomorphism in $\mathcal{M}$ which is a braided bialgebra homomorphism in $\mathcal{M}$ and let $i_{B^{\wedge_{E}}{ }^{n}}: B^{\wedge_{E} n} \rightarrow E$ and $i_{B^{\wedge_{E}}}^{{\wedge_{E}}^{b}}: B^{\wedge_{E} a} \rightarrow B^{\wedge_{E} b}(a \leq b)$ be the canonical injections. Assume that $i_{B^{\wedge} E^{a}}^{\wedge^{b}}$ is a split monomorphism in $\mathcal{M}$ for every $a \leq b$. The following assertions are equivalent.

(1) $g r_{B} E$ is the braided bialgebra of type one associated to $B$ and $\frac{B \wedge_{E} B}{B}$.

(2) $g r_{B} E$ is strongly $\mathbb{N}$-graded as an algebra.

(3) $A=\oplus_{n \in \mathbb{N}} B^{\wedge_{E} n+1}$ is strongly $\mathbb{N}$-graded as an algebra.

(4) $B^{\wedge_{E} n+1}=\left(B^{\wedge_{E} 2}\right)^{\cdot{ }^{*} n}$ for every $n \geq 2$.

Proof. Consider the graded algebra homomorphism

$$
\oplus_{n \in \mathbb{N}} p_{B^{\wedge} E^{n}}^{B^{\wedge} E^{n+1}}: \oplus_{n \in \mathbb{N}} B^{\wedge E} n+1 \rightarrow g r_{B} E
$$

of Proposition 4.5.

$(1) \Leftrightarrow(2)$ It follows in view of [AM1, Theorem 6.8] (where $A B 5$ is required) and by Theorem 2.10 .

$(2) \Leftrightarrow(3)$ It follows by Theorem 3.6 .

$(3) \Leftrightarrow(4)$ Let $\varphi: T=T_{B}\left(B^{\wedge_{E}}\right) \rightarrow \oplus_{n \in \mathbb{N}} B^{\wedge_{E}} n+1$ be the canonical morphism arising from the universal property of the tensor algebra and let $\varphi_{n}:\left(B^{\wedge_{E}}\right)^{\otimes_{B} n} \rightarrow B^{\wedge_{E} n+1}$ be its graded $n$-th component. In view of AM1, Theorem 3.11], (3) is equivalent to require that $\varphi_{n}$ is an epimorphism for every $n \geq 2$ (note that $\varphi_{0}$ and $\varphi_{1}$ are always isomorphisms). Let us prove that

$$
\varphi_{n} \circ \chi_{\left(B^{\wedge} E^{2}\right)^{\otimes_{B} n-1}, B^{\wedge} E^{2}}=m_{\wedge}^{n-1,1} \circ\left(\varphi_{n-1} \otimes B^{\wedge_{E} 2}\right) \text {, for every } n \geq 2 .
$$

where $\chi_{X, Y}: X \otimes Y \rightarrow X \otimes_{B} Y$ denotes the canonical projection.

Note that, being $\varphi$ a graded homomorphism and in view of its definition, one has

$$
i_{B^{\wedge} E^{n}}^{A} \circ \varphi_{n-1}=\varphi \circ i_{\left(B^{\wedge} E^{2}\right)^{\otimes_{B} n-1}}^{T}=\bar{m}_{A}^{n-2} \circ\left(i_{B^{\wedge} E^{2}}^{A}\right)^{\otimes_{B} n-1}
$$

so that

$$
\begin{aligned}
& \varphi_{n} \circ \chi_{\left(B^{\wedge} E^{2}\right)^{\otimes_{B}{ }^{n-1}, B^{\wedge} E^{2}}}=p_{n}^{A} \circ \bar{m}_{A}^{n-1} \circ\left(i_{B^{\wedge} E^{2}}^{A}\right)^{\otimes_{B} n} \circ \chi_{\left(B^{\wedge} E^{2}\right)^{\otimes_{B}{ }^{n-1}, B^{\wedge} E^{2}}} \\
= & p_{n}^{A} \circ m_{A} \circ\left[\left(\bar{m}_{A}^{n-2} \circ\left(i_{B^{\wedge} E^{2}}^{A}\right)\right.\right. \\
= & p_{n}^{A} \circ m_{A} \circ\left[\left(i_{B^{\wedge} E^{n}}^{A} \circ \varphi_{n-1}\right) \otimes i_{B^{\wedge} E^{2}}^{A}\right]=i_{B^{\wedge} E^{2}}^{A} \circ m_{A} \circ\left(i_{B^{\wedge} E^{n}}^{A} \otimes i_{B^{\wedge} E^{2}}^{A}\right) \circ\left(\varphi_{n-1} \otimes B^{\wedge_{E} 2}\right)
\end{aligned}
$$




$$
\text { 으 } p_{n}^{A} \circ i_{B^{\wedge} E^{n+1}}^{A} \circ m_{n-1,1}^{A} \circ\left(\varphi_{n-1} \otimes B^{\wedge_{E} 2}\right)=m_{\wedge}^{n-1,1} \circ\left(\varphi_{n-1} \otimes B^{\wedge_{E} 2}\right) \text {. }
$$

Hence 34 holds. Next we prove

$$
i_{B^{\wedge} E^{n+1}}^{E} \circ \varphi_{n}=\bar{m}_{E}^{n-1} \circ\left(i_{B^{\wedge} E^{2}}^{E}\right)^{\otimes_{B} n} \text {, for every } n \geq 2 .
$$

This is achieved by induction, composing on the right both sides with the epimorphism $\chi_{\left(B^{\wedge} E^{2}\right)^{\otimes B^{n-1}}, B^{\wedge} E^{2}}$ and using (34), (32).

Now, if $\varphi_{n}$ is an epimorphism, then

$$
\left(B^{\wedge_{E} n+1}, i_{B^{\wedge} E^{n+1}}^{E}\right)=\operatorname{Im}\left[\bar{m}_{E}^{n-1} \circ\left(i_{B^{\wedge_{E}}}^{E}\right)^{\otimes_{B} n}\right]=\left(B^{\wedge_{E} 2}\right)^{{ }_{E} n} .
$$

Conversely, if $\left(B^{\wedge_{E} n+1}, i_{B^{\wedge_{E}}{ }^{n+1}}\right)=\left(B^{\wedge_{E} 2}\right)^{{ }_{E} n}$, then there exists an epimorphism

$$
\varphi_{n}^{\prime}:\left(B^{\wedge_{E} 2}\right)^{\otimes_{B} n} \rightarrow B^{\wedge_{E} n+1}
$$

such that

$$
i_{B^{\wedge} E^{n+1}}^{E} \circ \varphi_{n}^{\prime}=\bar{m}_{E}^{n-1} \circ\left(i_{B^{\wedge} E^{2}}^{E}\right)^{\otimes_{B} n} .
$$

In view of (35) and since $i_{B^{\wedge} E^{n+1}}^{E}$ is a monomorphism, we get that $\varphi_{n}=\varphi_{n}^{\prime}$.

COROLlary 4.8. Let $H$ be a subbialgebra of a bialgebra $E$ over a field $K$. The following assertions are equivalent.

(1) $g r_{H} E$ is the bialgebra of type one associated to $H$ and $\frac{H \wedge_{E} H}{H}$.

(2) $g r_{H} E$ is strongly $\mathbb{N}$-graded as an algebra i.e. $g r_{H} E$ is generated as an algebra by $H$ and $\frac{H \wedge \wedge_{E} H}{H}$.

(3) $\bigoplus_{n \in \mathbb{N}} H^{\wedge_{E} n+1}$ is strongly $\mathbb{N}$-graded as an algebra.

(4) $H^{\wedge_{E} n+1}=\left(H \wedge_{E} H\right)^{\cdot{ }_{E}^{n}}$ for every $n \geq 2$.

Proof. We apply Theorem 4.7 to the case $(\mathcal{M}, c)=(\mathfrak{V e c}(K), \tau)$ where $\tau$ is the canonical flip.

REMARK 4.9. Let $H$ be a subbialgebra of a bialgebra $E$ over a field $K$ and assume that $H$ contains the coradical of $E$ (e.g. $E$ is connected). Assume that one of the conditions of Corollary 4.8 holds. Since $H$ contains the coradical of $E$, then the filtration $\left(H^{\wedge_{E} n+1}\right)_{n \in \mathbb{N}}$ is exhaustive hence (4) implies that $E$ is generated as an algebra by $H \wedge_{E} H$. The converse of this implication seems not to be true in general. Nevertheless we could not find a counterexample.

When $E$ is connected, in Corollary 4.8, we recover part of [Kh, Theorem 3.5] although for ordinary Hopf algebras.

\section{The Associated Graded Algebra of a Bialgebra With Respect to a Quotient BIALGEBRA}

LEMMA 5.1. Let $(\mathcal{M}, c)$ be a cocomplete coabelian braided monoidal category such that the tensor product commutes with direct sums. Consider in $(\mathcal{M}, c)$ a datum $\left(B, m_{B}, u_{B}, \Delta_{B}, \varepsilon_{B}\right)$ consisting of a graded object $B$ with graduation defined by $\left(B_{k}\right)_{k \in \mathbb{N}}$ such that, with respect to this graduation,

- $\left(B, m_{B}, u_{B}\right)$ is a graded algebra and

- $\left(B, \Delta_{B}, \varepsilon_{B}\right)$ is a graded coalgebra.

Assume that for every $a, b \in \mathbb{N}$,

$$
\begin{aligned}
& \sum_{s+t=a+b} \Delta_{a, b} m_{s, t}\left(p_{s} \otimes p_{t}\right) \\
&= \sum_{s^{\prime}+t^{\prime}=a} \sum_{s^{\prime \prime}+t^{\prime \prime}=b}\left(m_{s^{\prime}, t^{\prime}} \otimes m_{s^{\prime \prime}, t^{\prime \prime}}\right) \circ\left(B_{s^{\prime}} \otimes c_{B_{s^{\prime \prime}}, B_{t^{\prime}}} \otimes B_{t^{\prime \prime}}\right) \circ\left(\Delta_{s^{\prime}, s^{\prime \prime}} \otimes \Delta_{t^{\prime}, t^{\prime \prime}}\right) \circ\left(p_{s^{\prime}+s^{\prime \prime}} \otimes p_{t^{\prime}+t^{\prime \prime}}\right) \\
& \Delta_{0,0} \circ u_{0}=\left(u_{0} \otimes u_{0}\right) \circ \Delta_{\mathbf{1}} .
\end{aligned}
$$

Then $B$ is a graded braided bialgebra in $(\mathcal{M}, c)$.

Proof. It is analogous to that of Lemma 4.1 . 
Notations 5.2. From now on the following assumptions and notations will be used.

$(\mathcal{M}, c)$ is a cocomplete abelian coabelian braided monoidal category. Assume that the tensor product commutes with direct sums.

Let $\pi: E \rightarrow B$ be an epimorphism in $\mathcal{M}$ which is a braided bialgebra homomorphism in $\mathcal{M}$ and let $\left(I, i_{I}^{E}\right):=\operatorname{ker}(\pi)$. Assume that

$$
\frac{E}{i_{I^{a+1}}^{I^{a}}}: \frac{E}{I^{a+1}} \rightarrow \frac{E}{I^{a}}
$$

is a split epimorphism for every $a \in \mathbb{N}$, where $i_{I^{a+1}}^{I^{a}}: I^{a+1} \rightarrow I^{a}$ is the canonical injection.

The family $\left(\left(\frac{E}{I^{a}}\right)_{a \in \mathbb{N}},\left(\frac{E}{i_{I^{a}}^{I^{a}+1}}\right)_{a \in \mathbb{N}}\right)$ fulfills the conditions of Theorem 4.2 when regarded inside the dual of the abelian monoidal category $\mathcal{M}$. Thus we have the following exact sequence

$$
\bigoplus_{a+b=n} I^{a} \otimes I^{b} \stackrel{\nabla\left[\left(i_{I^{a}}^{E} \otimes i_{I^{b}}^{E}\right)_{a+b=n}\right]}{\longrightarrow} E \otimes E^{\Delta\left[\left(p_{I^{a}}^{E} \otimes p_{I^{b}}^{E}\right)_{a+b=n+1}\right]} \bigoplus_{a+b=n+1} \frac{E}{I^{a}} \otimes \frac{E}{I^{b}} .
$$

Let

and let

$$
\left(I_{n}(E), \beta_{n}\right):=\operatorname{Im}\left\{\Delta\left[\left(p_{I^{a}}^{E} \otimes p_{I^{b}}^{E}\right)_{a+b=n+1}\right]\right\}
$$

be the unique morphism such that

$$
\beta_{n} \circ \gamma_{n}=\Delta\left[\left(p_{I^{a}}^{E} \otimes p_{I^{b}}^{E}\right)_{a+b=n+1}\right] .
$$

Since $\left(I^{a+b}, i_{I^{a+b}}^{E}\right)=\operatorname{Im}\left(m_{E} \circ\left(i_{I^{a}}^{E} \otimes i_{I^{b}}^{E}\right)\right)$, we have $p_{I^{a+b}}^{E} \circ m_{E} \circ\left(i_{I^{a}}^{E} \otimes i_{I^{b}}^{E}\right)=0$ so that

$$
p_{I^{n}}^{E} \circ m_{E} \circ \nabla\left[\left(i_{I^{a}}^{E} \otimes i_{I^{b}}^{E}\right)_{a+b=n}\right]=0
$$

and hence, by the exactness of the sequence above, there exists a unique morphism

such that

$$
\alpha_{n}: I_{n}(E) \rightarrow \frac{E}{I^{n}}
$$

$$
p_{I^{n}}^{E} \circ m_{E}=\alpha_{n} \circ \gamma_{n}, \text { for every } n \in \mathbb{N} .
$$

Lemma 5.3. 0) For every $s, t, u, v \in \mathbb{N}$, we have

$$
\begin{aligned}
& \left(\beta_{u} \otimes \beta_{v}\right) \circ\left(\gamma_{u} \otimes \gamma_{v}\right) \circ(E \otimes c \otimes E) \circ\left(\Delta_{E} \otimes \Delta_{E}\right) \circ\left(i_{I^{s}}^{E} \otimes i_{I^{t}}^{E}\right) \\
= & \Delta\left[\left(\left(\frac{E}{I^{a}} \otimes c_{\frac{E}{I^{b}}, \frac{E}{I^{c}}} \otimes \frac{E}{I^{d}}\right) \circ\left(\left[\left(p_{I^{a}}^{E} \otimes p_{I^{b}}^{E}\right) \Delta_{E} i_{I^{s}}^{E}\right] \otimes\left[\left(p_{I^{c}}^{E} \otimes p_{I^{d}}^{E}\right) \Delta_{E} i_{I^{t}}^{E}\right]\right)\right)_{\substack{a+c=u+1 \\
b+d=v+1}}\right]
\end{aligned}
$$

1) The following relations hold.

$$
\left(p_{I^{u}}^{E} \otimes p_{I^{v}}^{E}\right) \circ \Delta_{E} \circ i_{I^{u+v-1}}^{E}=0 \text { for every } u, v \in \mathbb{N}, u+v \geq 1 .
$$

2) For every $a, b \in \mathbb{N}$, there exists a unique morphism

such that

$$
\Delta_{\vee}^{a, b}: \frac{E}{I^{a+b+1}} \rightarrow \frac{E}{I^{a+1}} \otimes \frac{E}{I^{b+1}}
$$

$$
\Delta_{\vee}^{a, b} \circ p_{I^{a+b+1}}^{E}=\left(p_{I^{a+1}}^{E} \otimes p_{I^{b+1}}^{E}\right) \Delta_{E}, \text { for every } a, b \in \mathbb{N} .
$$

3) For every $a, b, c, d \in \mathbb{N}$, we have

$$
\begin{gathered}
\left(\Delta_{\vee}^{a, b} \otimes \frac{E}{I^{c+1}}\right) \circ \Delta_{\vee}^{a+b, c}=\left(\frac{E}{I^{a+1}} \otimes \Delta_{\vee}^{b, c}\right) \circ \Delta_{\vee}^{a, b+c}, \\
\left(\frac{E}{I^{d+1}} \otimes \varepsilon_{B}\right) \circ \Delta_{\vee}^{d, 0}=r_{E / I^{d+1}}^{-1}, \quad\left(\varepsilon_{B} \otimes \frac{E}{I^{d+1}}\right) \circ \Delta_{\vee}^{0, d}=l_{E / I^{d+1}}^{-1},
\end{gathered}
$$




$$
\left(\frac{E}{i_{I^{a+2}}^{I^{a+1}}} \otimes \frac{E}{I^{b+1}}\right) \circ \Delta_{\vee}^{a+1, b}=\Delta_{\vee}^{a, b} \circ \frac{E}{i_{I^{a+b+2}}^{I^{a+b+1}}}, \quad\left(\frac{E}{I^{a+1}} \otimes \frac{E}{i_{I^{b+2}}^{I^{b+1}}}\right) \circ \Delta_{\vee}^{a, b+1}=\Delta_{\vee}^{a, b} \circ \frac{E}{i_{I^{a+b+2}}^{I^{a+b+1}}}
$$

Proof. It is analogous to that of Lemma 4.4.

Proposition 5.4. $\oplus_{n \in \mathbb{N}} \frac{E}{I^{n+1}}$ is a graded coalgebra and there is a unique coalgebra structure on $g r_{I} E=\oplus_{n \in \mathbb{N}} g r_{I}^{n} E$ such that

$$
\oplus_{n \in \mathbb{N}} \frac{i_{I^{n}}^{E}}{I^{n+1}}: g r_{I} E \rightarrow \oplus_{n \in \mathbb{N}} \frac{E}{I^{n+1}}
$$

is a coalgebra homomorphism and

(1) $\operatorname{gr}_{I} E=\oplus_{n \in \mathbb{N}} g r_{I}^{n} E$ is a graded coalgebra such that $\oplus_{n \in \mathbb{N}} \frac{i_{I n}^{E}}{I^{n+1}}$ is a graded homomorphism;

$$
\left(\frac{i_{I^{a}}^{E}}{I^{a+1}} \otimes \frac{i_{I^{b}}^{E}}{I^{b+1}}\right) \circ \Delta_{a, b}^{g r_{I} E}=\Delta_{\vee}^{a, b} \circ \frac{i_{I^{a+b}}^{E}}{I^{a+b+1}}
$$

(3) $\varepsilon_{g r_{I} E}=\varepsilon_{B} \circ p_{0}^{g r_{I} E}$.

Proof. By Lemma 5.3, we can apply the Lemma 2.5 to the family $\left(\frac{E}{I^{n+1}}\right)_{n \in \mathbb{N}}$.

THEOREM 5.5. Let $(\mathcal{M}$, c) be a cocomplete and complete abelian coabelian braided monoidal category satisfying AB5. Assume that the tensor product commutes with direct sums.

Let $\pi: E \rightarrow B$ be an epimorphism in $\mathcal{M}$ which is a braided bialgebra homomorphism in $\mathcal{M}$ and let $\left(I, i_{I}^{E}\right):=\operatorname{ker}(\pi)$. Assume that

$$
\frac{E}{i_{I^{a+1}}^{I^{a}}}: \frac{E}{I^{a+1}} \rightarrow \frac{E}{I^{a}}
$$

is a split epimorphism for every $a \in \mathbb{N}$, where $i_{I^{a+1}}^{I^{a}}: I^{a+1} \rightarrow I^{a}$ is the canonical injection.

Then $\mathrm{gr}_{I} E$ is a graded braided bialgebra in $(\mathcal{M}, c)$.

Proof. It is analogous to that of Theorem 4.6.

TheOREM 5.6. Let $(\mathcal{M}, c)$ be a cocomplete and complete abelian coabelian braided monoidal category satisfying AB5. Assume that the tensor product commutes with direct sums. Let $\pi: E \rightarrow B$ be an epimorphism in $\mathcal{M}$ which is a braided bialgebra homomorphism in $\mathcal{M}$ and let $\left(I, i_{I}^{E}\right):=\operatorname{ker}(\pi)$. Assume that

$$
\frac{E}{i_{I^{a+1}}^{I^{a}}}: \frac{E}{I^{a+1}} \rightarrow \frac{E}{I^{a}}
$$

is a split epimorphism for every $a \in \mathbb{N}$, where $i_{I^{a+1}}^{I^{a}}: I^{a+1} \rightarrow I^{a}$ is the canonical injection. The following assertions are equivalent.

(1) $g r_{I} E$ is the braided bialgebra of type one associated to $B=\frac{E}{I}$ and $\frac{I}{I^{2}}$.

(2) $g_{I} E$ is strongly $\mathbb{N}$-graded as a coalgebra.

(3) $C=\oplus_{n \in \mathbb{N}} \frac{E}{I^{n+1}}$ is strongly $\mathbb{N}$-graded as a coalgebra.

(4) $\left(I^{n+1}, i_{I^{n+1}}^{E}\right)=\left(I^{2}\right)^{\wedge_{E} n}$ for every $n \geq 2$.

Proof. Consider the graded coalgebra homomorphism

$$
\oplus_{n \in \mathbb{N}} \frac{i_{I^{n}}^{E}}{I^{n+1}}: g r_{I} E \rightarrow \oplus_{n \in \mathbb{N}} \frac{E}{I^{n+1}}
$$

of Proposition 5.4. Note that in view of $A B 5$ condition, this morphism is indeed a monomorphism. 3.10 .

$(1) \Leftrightarrow(2)$ It follows in view of [AM1, Theorem 6.8] (where $A B 5$ is required) and by Theorem

$(2) \Leftrightarrow(3)$ It follows by Theorem 2.7 that, by Lemma 5.3, can be applied to the family $\left(\frac{E}{I^{n+1}}\right)_{n \in \mathbb{N}}$.

$(3) \Leftrightarrow(4)$ Let $\psi: \oplus_{n \in \mathbb{N}} \frac{E}{I^{n+1}} \rightarrow T^{c}=T_{\frac{E}{T}}^{c}\left(\frac{E}{I^{2}}\right)$ be the canonical morphism arising from the universal property of the tensor algebra and let

$$
\psi_{n}: \frac{E}{I^{n+1}} \rightarrow\left(\frac{E}{I^{2}}\right)^{\square_{B} n}
$$


be its graded $n$-th component. In view of AM1, Theorem 2.22], (3) is equivalent to require that $\psi_{n}$ is an epimorphism for every $n \geq 2$ (note that $\psi_{0}$ and $\psi_{1}$ are always isomorphisms). Let us prove that

$$
\zeta_{\left(\frac{E}{I^{2}}\right)^{\square_{B} n-1}, \frac{E}{I^{2}}} \circ \psi_{n}=\left(\psi_{n-1} \otimes \frac{E}{I^{2}}\right) \circ \Delta_{\vee}^{n-1,1}, \text { for every } n \geq 2
$$

where $\zeta_{X, Y}: X \square_{B} Y \rightarrow X \otimes Y$ denotes the canonical injection.

Note that, being $\psi$ a graded homomorphism and in view of [AM1, Theorem 2.16 and Proposition 2.19], one has

so that

$$
\psi_{n} \circ p_{n-1}^{C}=p_{n-1}^{T^{c}} \circ \psi=\left(p_{1}^{C}\right)^{\square_{B} n-1} \circ \bar{\Delta}_{C}^{n-2}
$$

$$
\begin{aligned}
& \zeta_{\left(\frac{E}{I^{2}}\right)^{\square_{B} n-1}, \frac{E}{I^{2}}} \circ \psi_{n} \\
= & \zeta_{\left(\frac{E}{I^{2}}\right)^{\square_{B} n-1}, \frac{E}{I^{2}}} \circ\left(p_{1}^{C}\right)^{\square_{B} n} \circ \bar{\Delta}_{C}^{n-1} \circ i_{n}^{C}=\left[\left(p_{1}^{C}\right)^{\square_{B} n-1} \circ \bar{\Delta}_{C}^{n-2} \otimes p_{1}^{C}\right] \circ \Delta_{C} \circ i_{n}^{C} \\
= & {\left[\left(\psi_{n-1} \circ p_{n-1}^{C}\right) \otimes p_{1}^{C}\right] \circ \Delta_{C} \circ i_{n}^{C}=\left(\psi_{n-1} \otimes C_{1}\right) \circ\left(p_{n-1}^{C} \otimes p_{1}^{C}\right) \circ \Delta_{C} \circ i_{n}^{C} } \\
& \underline{\underline{D}}\left(\psi_{n-1} \otimes \frac{E}{I^{2}}\right) \circ \Delta_{n-1,1}^{C} \circ p_{n}^{C} \circ i_{n}^{C}=\left(\psi_{n-1} \otimes \frac{E}{I^{2}}\right) \circ \Delta_{\vee}^{n-1,1} .
\end{aligned}
$$

Hence 42 holds. Let us prove by induction that

$$
\psi_{n} \circ p_{I^{n+1}}^{E}=\left(p_{I^{2}}^{E}\right)^{\square_{B} n} \circ \bar{\Delta}_{E}^{n-1}, \text { for every } n \geq 2 \text {. }
$$

$n=2$ ) We have

$$
\begin{aligned}
& \zeta_{\frac{E}{I^{2}}, \frac{E}{I^{2}}} \circ \psi_{2} \circ p_{I^{3}}^{E} \stackrel{42)}{=}\left(\psi_{1} \otimes \frac{E}{I^{2}}\right) \circ \Delta_{\vee}^{1,1} \circ p_{I^{3}}^{E} \\
= & \Delta_{\vee}^{1,1} \circ p_{I^{3}}^{E} \stackrel{(40)}{=}\left(p_{I^{2}}^{E} \otimes p_{I^{2}}^{E}\right) \circ \Delta_{E}=\zeta_{\frac{E}{I^{2}}, \frac{E}{I^{2}}} \circ\left(p_{I^{2}}^{E} \square_{B} p_{I^{2}}^{E}\right) \circ \bar{\Delta}_{E}
\end{aligned}
$$

$n-1 \Rightarrow n)$ We have

$$
\begin{aligned}
& \zeta_{\left(\frac{E}{I^{2}}\right)^{\square_{B} n-1, \frac{E}{I^{2}}}} \circ \psi_{n} \circ p_{I^{n+1}}^{E} \stackrel{\text { 年) }}{=}\left(\psi_{n-1} \otimes \frac{E}{I^{2}}\right) \circ \Delta_{\vee}^{n-1,1} \circ p_{I^{n+1}}^{E} \\
& \stackrel{(40)}{=}\left(\psi_{n-1} \otimes \frac{E}{I^{2}}\right) \circ\left(p_{I^{n}}^{E} \otimes p_{I^{2}}^{E}\right) \circ \Delta_{E} \\
= & \left(\left(p_{I^{2}}^{E}\right)^{\square_{B} n-1} \otimes p_{I^{2}}^{E}\right) \circ\left(\bar{\Delta}_{E}^{n-2} \otimes \frac{E}{I^{2}}\right) \circ \Delta_{E}=\zeta_{\left(\frac{E}{I^{2}}\right)^{\square_{B}{ }^{n-1}, \frac{E}{I^{2}}}} \circ\left(p_{I^{2}}^{E}\right)^{\square_{B} n} \circ \bar{\Delta}_{E}^{n-1} .
\end{aligned}
$$

We have so proved that (43) holds.

Now, if $\psi_{n}$ is a monomorphism, then

$$
\left(I^{n+1}, i_{I^{n+1}}^{E}\right)=\operatorname{ker}\left(\psi_{n} \circ p_{I^{n+1}}^{E}\right)=\operatorname{ker}\left[\left(p_{I^{2}}^{E}\right)^{\square_{B} n} \circ \bar{\Delta}_{E}^{n-1}\right]=\operatorname{ker}\left[\left(p_{I^{2}}^{E}\right)^{\otimes n} \circ \Delta_{E}^{n-1}\right]=\left(I^{2}\right)^{\wedge_{E} n} .
$$

Conversely, if $\left(I^{n+1}, i_{I^{n+1}}^{E}\right)=\left(I^{2}\right)^{\wedge_{E} n}$, then $\left(p_{I^{2}}^{E}\right)^{\square_{B} n} \circ \bar{\Delta}_{E}^{n-1}$ factors to a monomorphism

$$
\psi_{n}^{\prime}: \frac{E}{I^{n+1}} \rightarrow\left(\frac{E}{I^{2}}\right)^{\square_{B} n}
$$

such that $\psi_{n}^{\prime} \circ p_{I^{n+1}}^{E}=\left(p_{I^{2}}^{E}\right)^{\square_{B} n} \circ \bar{\Delta}_{E}^{n-1}$. In view of (43) and since $p_{I^{n+1}}^{E}$ is an epimorphism, we get that $\psi_{n}=\psi_{n}^{\prime}$.

COROLlary 5.7. Let $J$ an ideal of a bialgebra $E$ over a field $K$ and assume that $J$ is also a coideal. The following assertions are equivalent.

(1) $g r_{J} E$ is the bialgebra of type one associated to $\frac{E}{J}$ and $\frac{J}{J^{2}}$.

(2) $\operatorname{gr}_{J} E$ is strongly $\mathbb{N}$-graded as a coalgebra.

(3) $\oplus_{n \in \mathbb{N}} \frac{E}{J^{n+1}}$ is strongly $\mathbb{N}$-graded as a coalgebra.

(4) $J^{n+1}=\left(J^{2}\right)^{\wedge}$ for every $n \geq 2$. 
Proof. We apply Theorem 5.6 to the case $(\mathcal{M}, c)=(\mathfrak{V} \mathfrak{e c}(K), \tau)$ where $\tau$ is the canonical flip.

\section{Appendix A. TeChniCAlities}

Proof of Lemma 4.4. Set $i_{n}:=i_{B^{\wedge} E^{a}}^{E}$ and $p_{n}:=p_{B^{\wedge} E^{n}}^{E}$.

0 ) It follows by using (28) and naturality of $c$.

1) Let us prove by induction on $u+v \geq 1$ that $p_{u+v-1} m_{E}\left(i_{u} \otimes i_{v}\right)=0$.

$u+v=1)$ is trivial as $i_{0}=0$.

$u+v>1$ ) If $u=0$ or $v=0$ there is nothing to prove. Let $u, v>0$. Assume that the statement is true for every $i, j$ such that $1 \leq i+j<u+v$ and let us prove it for $u+v$. First of all we will prove that

$$
\left(p_{u-1} \otimes p_{v}\right) \circ \Delta_{E} \circ m_{E} \circ\left(i_{u} \otimes i_{v}\right)=0 .
$$

Using the compatibility of $\Delta_{E}$ and $m_{E}$, and (29) we get

$$
\begin{aligned}
& \left(p_{u-1} \otimes p_{v}\right) \circ \Delta_{E} \circ m_{E} \circ\left(i_{u} \otimes i_{v}\right) \\
= & \left(p_{u-1} \otimes p_{v}\right) \circ\left(m_{E} \otimes m_{E}\right) \circ(E \otimes c \otimes E) \circ\left(\beta_{u} \otimes \beta_{v}\right) \circ\left(\alpha_{u} \otimes \alpha_{v}\right)
\end{aligned}
$$

Let us prove that

$$
\left(p_{u-1} \otimes p_{v}\right) \circ\left(m_{E} \otimes m_{E}\right) \circ(E \otimes c \otimes E) \circ\left(\beta_{u} \otimes \beta_{v}\right)=0 .
$$

Since $\gamma_{u} \otimes \gamma_{v}$ is an epimorphism, this is equivalent to prove that

$$
\left(p_{u-1} \otimes p_{v}\right) \circ\left(m_{E} \otimes m_{E}\right) \circ(E \otimes c \otimes E) \circ\left(\beta_{u} \otimes \beta_{v}\right) \circ\left(\gamma_{u} \otimes \gamma_{v}\right)=0 .
$$

We have

$$
\begin{aligned}
& \left(p_{u-1} \otimes p_{v}\right) \circ\left(m_{E} \otimes m_{E}\right) \circ(E \otimes c \otimes E) \circ\left(\beta_{u} \otimes \beta_{v}\right) \circ\left(\gamma_{u} \otimes \gamma_{v}\right) \\
& \stackrel{\text { (30) }}{=} \nabla\left[\left(\left(\left[p_{u-1} m_{E}\left(i_{a} \otimes i_{b}\right)\right] \otimes\left[p_{v} m_{E}\left(i_{c} \otimes i_{d}\right)\right]\right) \circ\left(B^{\wedge_{E} a} \otimes c_{B^{\wedge^{c}}, B^{\wedge} E^{b}} \otimes B^{\wedge_{E} d}\right)\right)_{\substack{a+c=u+1 \\
b+d=v+1}}\right]
\end{aligned}
$$

Note that $(a+b)+(c+d)=(a+c)+(b+d)=u+v+2$.

If $u-1 \geq a+b-1$ then $a+b \leq u<u+v$ and we have

$$
p_{u-1} m_{E}\left(i_{a} \otimes i_{b}\right)=\frac{E}{\xi_{a+b-1}^{u-1}} p_{a+b-1} m_{E}\left(i_{a} \otimes i_{b}\right)=0 .
$$

If $u-1<a+b-1$, and $v<c+d-1$, then $u+v \leq a+b+c+d-3=u+v-1$. A contradiction.

Then $v \geq c+d-1$. Thus $c+d<v \leq u+v$ so that, as above, we get $p_{v} m_{E}\left(i_{c} \otimes i_{d}\right)=0$.

Hence

$$
\left(p_{u-1} \otimes p_{v}\right) \circ\left(m_{E} \otimes m_{E}\right) \circ(E \otimes c \otimes E) \circ\left(\beta_{u} \otimes \beta_{v}\right) \circ\left(\gamma_{u} \otimes \gamma_{v}\right)=0
$$

and so (44) holds.

Let $\Delta_{u-1, v}:=\Delta_{B^{\wedge} E^{u-1}, B^{\wedge} E^{v}}=\left(p_{u-1} \otimes p_{v}\right) \circ \Delta_{E}$. Then, as seen in 2.8, there exists a unique morphism

$$
\bar{\Delta}_{u-1, v}: \frac{E}{B^{\wedge_{E} u+v-1}}=\frac{E}{B^{\wedge_{E} u-1} \wedge_{E} B^{\wedge_{E} v}} \rightarrow \frac{E}{B^{\wedge_{E} u-1}} \otimes \frac{E}{B^{\wedge_{E} v}}
$$

such that $\bar{\Delta}_{u-1, v} \circ p_{u+v-1}=\Delta_{u-1, v}$. Furthermore $\bar{\Delta}_{u-1, v}$ is a monomorphism. From

$$
\bar{\Delta}_{u-1, v} \circ\left[p_{u+v-1} \circ m_{E} \circ\left(i_{u} \otimes i_{v}\right)\right]=\Delta_{u-1, v} \circ m_{E} \circ\left(i_{u} \otimes i_{v}\right) \stackrel{(44)}{=} 0
$$

we deduce $p_{u+v-1} \circ m_{E} \circ\left(i_{u} \otimes i_{v}\right)=0$ so that we have proved (31).

2) From (31), we get $p_{a+b+1} m_{E}\left(i_{a+1} \otimes i_{b+1}\right)=0$ for every $a, b \in \mathbb{N}$. By the universal property of the kernel there exists a unique morphism $m_{\wedge}^{a, b}: B^{\wedge_{E} a+1} \otimes B^{\wedge_{E} b+1} \rightarrow B^{\wedge_{E} a+b+1}$ such that (32) holds.

3) From (32), we get

$$
\begin{aligned}
& i_{a+b+c+1} \circ m_{\wedge}^{a+b, c} \circ\left(m_{\wedge}^{a, b} \otimes B^{\wedge_{E} c+1}\right)=m_{E}\left(m_{E} \otimes E\right)\left(i_{a+1} \otimes i_{b+1} \otimes i_{c+1}\right) \\
& i_{a+b+c+1} \circ m_{\wedge}^{a, b+c} \circ\left(B^{\wedge_{E} a+1} \otimes m_{\wedge}^{b, c}\right)=m_{E}\left(E \otimes m_{E}\right)\left(i_{a+1} \otimes i_{b+1} \otimes i_{c+1}\right)
\end{aligned}
$$


so that, by associativity of $m_{E}$, we obtain

$$
i_{a+b+c+1} \circ m_{\wedge}^{a+b, c} \circ\left(m_{\wedge}^{a, b} \otimes B^{\wedge_{E} c+1}\right)=i_{a+b+c+1} \circ m_{\wedge}^{a, b+c} \circ\left(B^{\wedge_{E} a+1} \otimes m_{\wedge}^{b, c}\right) .
$$

Since $i_{a+b+c+1}$ is a monomorphism, we deduce that

$$
m_{\wedge}^{a+b, c} \circ\left(m_{\wedge}^{a, b} \otimes B^{\wedge_{E} c+1}\right)=m_{\wedge}^{a, b+c} \circ\left(B^{\wedge_{E} a+1} \otimes m_{\wedge}^{b, c}\right) .
$$

On the other hand, by applying (32), we infer that

$$
i_{d+1} \circ m_{\wedge}^{d, 0} \circ\left(B^{\wedge_{E} d+1} \otimes u_{B}\right)=m_{E}\left(E \otimes u_{E}\right)\left(i_{d+1} \otimes \mathbf{1}\right)=r_{E}\left(i_{d+1} \otimes \mathbf{1}\right)=i_{d+1} \circ r_{B^{\wedge} E^{d+1}}
$$

so that, since $i_{d+1}$ is a monomorphism, we obtain $m_{\wedge}^{d, 0} \circ\left(B^{\wedge_{E} d+1} \otimes u_{B}\right)=r_{B^{\wedge} E^{d+1}}$. Similarly we prove that $m_{\wedge}^{0, d} \circ\left(u_{B} \otimes B^{\wedge_{E} d+1}\right)=l_{B^{\wedge} E^{d+1}}$. We have

$$
\begin{aligned}
& i_{a+b+2} \circ m_{\wedge}^{a+1, b} \circ\left(i_{B^{\wedge^{\prime}} B^{a+1}}^{{\wedge^{a+2}}^{a+2}} \otimes B^{\wedge_{E} b+1}\right)=m_{E} \circ\left(i_{a+2} \otimes i_{b+1}\right) \circ\left(i_{B^{\wedge E^{a+1}}}^{{\wedge^{a+2}}^{a+2}} \otimes B^{\wedge_{E} b+1}\right) \\
= & m_{E} \circ\left(i_{a+1} \otimes i_{b+1}\right)=i_{a+b+1} \circ m_{\wedge}^{a, b}=i_{a+b+2} \circ i_{B^{\wedge_{E}}}^{{\wedge^{a+b+1}}^{a+b+2}} \circ m_{\wedge}^{a, b} .
\end{aligned}
$$

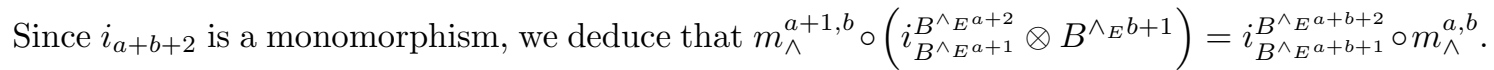
The right hand version of this formula follows by similar arguments.

LEMma A.1. There exists a unique morphism

$$
\theta_{a, b}: \sum_{u+v=a+b+2} B^{\wedge_{E} u} \otimes B^{\wedge_{E} v} \rightarrow \frac{B^{\wedge_{E} a+1}}{B^{\wedge_{E} a}} \otimes \frac{B^{\wedge_{E} b+1}}{B^{\wedge_{E} b}}
$$

such that

$$
\left(p_{B^{\wedge} E^{a}}^{B^{\wedge} E^{a+1}} \otimes p_{B^{\wedge^{\wedge}}}^{B^{\wedge} E^{b+1}}\right) \circ \beta_{a+b+1}=\left(\frac{i_{B^{\wedge_{E}} a+1}^{E}}{B^{\wedge_{E} a}} \otimes \frac{i_{B^{\wedge} E^{b+1}}^{E}}{B^{\wedge_{E} b}}\right) \circ \theta_{a, b} .
$$

Moreover, for every $a, b \in \mathbb{N}$, we have

$$
\begin{gathered}
\theta_{a, b} \circ \alpha_{a+b+1}=\Delta_{a, b}^{g r_{B} E} \circ p_{B^{\wedge^{\wedge}} E^{a+b}}^{{\wedge^{a+b+1}}^{a+b}}, \\
\theta_{a, b} \circ \gamma_{a+b+1}=\nabla\left[\left(\delta_{u, a+1} \delta_{v, b+1} p_{B^{\wedge} E^{a+1}}^{{\wedge^{a}}^{a+1}} \otimes p_{B^{\wedge} E^{b}}^{{\wedge^{\prime} E^{b+1}}^{b}}\right)_{u+v=a+b+2}\right] .
\end{gathered}
$$

Proof. Set $i_{n}:=i_{B^{\wedge^{a}} a}^{E}, p_{n}:=p_{B^{\wedge} E^{n}}^{E}$ and $p_{n}^{n+1}:=p_{B^{\wedge E^{n}}}^{{\wedge^{n}}^{n+1}}$.

Since $p_{s} i_{t}=0$ for every $s \geq t$, we have

$$
\left(\frac{E}{B^{\wedge_{E}} a} \otimes \frac{E}{\xi_{b}^{b+1}}\right) \circ\left(p_{a} \otimes p_{b}\right) \circ \beta_{a+b+1} \circ \gamma_{a+b+1} \stackrel{(28)}{=} \nabla\left[\left[\left(p_{a} i_{u} \otimes p_{b+1} i_{v}\right)\right]_{u+v=a+b+2}\right]=0 .
$$

Since $\gamma_{a+b+1}$ is an epimorphism, we get

$$
\left(\frac{E}{B^{\wedge_{E} a}} \otimes \frac{E}{\xi_{b}^{b+1}}\right) \circ\left(p_{a} \otimes p_{b}\right) \circ \beta_{a+b+1}=0 .
$$

By the universal property of the kernel applied to the exact sequence,

$$
0 \rightarrow \frac{E}{B^{\wedge_{E} a}} \otimes \frac{B^{\wedge_{E} b+1}}{B^{\wedge_{E} b}} \stackrel{\frac{E}{B^{\wedge} E^{a}} \otimes}{\longrightarrow} \frac{i_{b+1}}{B^{\wedge^{b}}} \frac{E}{B^{\wedge_{E} a}} \otimes \frac{E}{B^{\wedge_{E} b}} \stackrel{\frac{E}{B^{\Lambda} a} \otimes \frac{E}{\xi_{b}^{b+1}}}{\longrightarrow} \frac{E}{B^{\wedge_{E} a}} \otimes \frac{E}{B^{\wedge_{E} b+1}} \rightarrow 0 .
$$

there exists a unique morphism

$$
\theta_{a, b}^{\prime}: \sum_{u+v=a+b+2} B^{\wedge_{E} u} \otimes B^{\wedge_{E} v} \rightarrow \frac{E}{B^{\wedge_{E} a}} \otimes \frac{B^{\wedge_{E} b+1}}{B^{\wedge_{E} b}}
$$

such that

$$
\left(\frac{E}{B^{\wedge} a} \otimes \frac{i_{b+1}}{B^{\wedge_{E}} b}\right) \circ \theta_{a, b}^{\prime}=\left(p_{a} \otimes p_{b}\right) \circ \beta_{a+b+1} .
$$


Now

$$
\left(\frac{E}{B^{\wedge_{E} a+1}} \otimes \frac{i_{b+1}}{B^{\wedge_{E} b}}\right) \circ\left(\frac{E}{\xi_{a}^{a+1}} \otimes \frac{B^{\wedge_{E} b+1}}{B^{\wedge_{E} b}}\right) \circ \theta_{a, b}^{\prime}=\left(\frac{E}{\xi_{a}^{a+1}} \otimes \frac{E}{B^{\wedge_{E} b}}\right) \circ\left(p_{a} \otimes p_{b}\right) \circ \beta_{a+b+1}=0
$$

where the last equality follows analogously to 48 . Since $\frac{E}{B^{\wedge} E^{a+1}} \otimes \frac{i_{b+1}}{B^{\wedge} E^{b}}$ is a monomorphism we get $\left(\frac{E}{\xi_{a}^{a+1}} \otimes \frac{B^{\wedge} E^{b+1}}{B^{\wedge} E^{b}}\right) \circ \theta_{a, b}^{\prime}=0$. By the universal property of the kernel applied to the exact
sequence,

$$
0 \rightarrow \frac{B^{\wedge_{E} a+1}}{B^{\wedge_{E}} a} \otimes \frac{B^{\wedge_{E} b+1}}{B^{\wedge_{E} b}} \frac{i_{a+1}}{B^{\wedge_{E}}} \stackrel{\otimes \frac{B^{\wedge} E_{E} b+1}{B^{\wedge} E^{b}}}{B^{\wedge_{E} a}} \otimes \frac{E}{B^{\wedge_{E} b}} \stackrel{B^{\wedge_{E} b+1}}{\xi_{a}^{a+1} \otimes \frac{B^{\wedge} E^{b+1}}{B^{\wedge} E^{b}}} \frac{E}{B^{\wedge_{E} a+1}} \otimes \frac{B^{\wedge_{E} b+1}}{B^{\wedge_{E} b}} .
$$

there exists a unique morphism

$$
\theta_{a, b}: \sum_{u+v=a+b+2} B^{\wedge_{E} u} \otimes B^{\wedge_{E} v} \rightarrow \frac{B^{\wedge_{E} a+1}}{B^{\wedge_{E} a}} \otimes \frac{B^{\wedge_{E} b+1}}{B^{\wedge_{E} b}}
$$

such that

$$
\left(\frac{i_{a+1}}{B^{\wedge_{E} a}} \otimes \frac{B^{\wedge_{E} b+1}}{B^{\wedge_{E} b}}\right) \circ \theta_{a, b}=\theta_{a, b}^{\prime}
$$

Thus (45) holds true.

Let us prove 46 ). We have

$$
\left(\frac{i_{a+1}}{B^{\wedge_{E} a}} \otimes \frac{i_{b+1}}{B^{\wedge_{E} b}}\right) \circ \theta_{a, b} \circ \alpha_{a+b+1}=\left(p_{a} \otimes p_{b}\right) \circ \beta_{a+b+1} \circ \alpha_{a+b+1}=\left(p_{a} \otimes p_{b}\right) \circ \Delta_{E} \circ i_{a+b+1} .
$$

On the other hand, in view of definition of $\Delta_{a, b}^{B}$ (see 2.8), we have

$$
\begin{aligned}
& \left(\frac{i_{a+1}}{B^{\wedge} a} \otimes \frac{i_{b+1}}{B^{\wedge} b}\right) \circ \Delta_{a, b}^{g r_{B} E} \circ p_{a+b}^{a+b+1} \stackrel{\sqrt{16}}{=} \Delta_{a, b}^{B} \circ \frac{i_{a+b+1}}{B^{\wedge}{ }_{E} a+b} \circ p_{a+b}^{a+b+1} \\
= & \Delta_{a, b}^{B} \circ p_{a+b} \circ i_{a+b+1}=\left(p_{a} \otimes p_{b}\right) \circ \Delta_{E} \circ i_{a+b+1} .
\end{aligned}
$$

Hence

$$
\left(\frac{i_{a+1}}{B^{\wedge_{E} a}} \otimes \frac{i_{b+1}}{B^{\wedge_{E} b}}\right) \circ \theta_{a, b} \circ \alpha_{a+b+1}=\left(\frac{i_{a+1}}{B^{\wedge_{E} a}} \otimes \frac{i_{b+1}}{B^{\wedge_{E} b}}\right) \circ \Delta_{a, b}^{g r_{B} E} \circ p_{a+b}^{a+b+1} .
$$

Since $\frac{i_{a+1}}{B^{\wedge} E^{a}} \otimes \frac{i_{b+1}}{B^{\wedge} E^{b}}$ is a monomorphism, we get (46).

Let us prove 47 ). We have

$$
\begin{aligned}
& \left(\frac{i_{a+1}}{B^{\wedge_{E}} a} \otimes \frac{i_{b+1}}{B^{\wedge_{E} b}}\right) \circ \theta_{a, b} \circ \gamma_{a+b+1} \stackrel{45}{=}\left(p_{a} \otimes p_{b}\right) \circ \beta_{a+b+1} \circ \gamma_{a+b+1} \\
& \text { 22) }\left(p_{a} \otimes p_{b}\right) \circ \nabla\left[\left(i_{u} \otimes i_{v}\right)_{u+v=a+b+2}\right]=\nabla\left[\left(p_{a} i_{u} \otimes p_{b} i_{v}\right)_{u+v=a+b+2}\right] \\
& =\nabla\left[\delta_{u, a+1} \delta_{v, b+1}\left(p_{a} i_{a+1} \otimes p_{b} i_{b+1}\right)_{u+v=a+b+2}\right] \\
& =\left(\frac{i_{a+1}}{B^{\wedge_{E} a}} \otimes \frac{i_{b+1}}{B^{\wedge} b}\right) \circ \nabla\left[\delta_{u, a+1} \delta_{v, b+1}\left(p_{a}^{a+1} \otimes p_{b}^{b+1}\right)_{u+v=a+b+2}\right] \text {. }
\end{aligned}
$$

Since $\frac{i_{a+1}}{B^{\wedge} E^{a}} \otimes \frac{i_{b+1}}{B^{\wedge} E^{b}}$ is a monomorphism we get $(47)$.

\section{REFERENCES}

[AM1] A. Ardizzoni and C. Menini, Braided Bialgebras of Type One, Comm. Algebra, Vol. 36(11) (2008), 42964337.

[AM2] A. Ardizzoni and C. Menini, Some Remarks on Connected Coalgebras, Algebr. Represent. Theory, Vol. 12 (2009), 235-249.

[AM3] A. Ardizzoni and C. Menini, A Categorical Proof of a Useful Result, in "Modules and Comodules" Proceedings of a conference dedicated to Robert Wisbauer. Edited by T. Breziński, J. L. Gómez Pardo, I. Shestakov and P. F. Smith. Trends in Math Vol. XII, Birkhäuser Verlag, Basel, 2008, 31-45.

[AMS1] A. Ardizzoni, C. Menini and D. Ştefan, Cotensor Coalgebras in Monoidal Categories, Comm. Algebra, Vol. 35, N. 1 (2007), 25-70.

[AMS2] A. Ardizzoni, C. Menini and D. Ştefan, Hochschild Cohomology And 'Smoothness' In Monoidal Categories, J. Pure Appl. Algebra, 208 (2007), 297-330. 
[AS] N. Andruskiewitsch and H-J. Schneider, Pointed Hopf algebras. New directions in Hopf algebras, 1-68, Math. Sci. Res. Inst. Publ., 43, Cambridge Univ. Press, Cambridge, 2002.

[Ka] C. Kassel, Quantum Groups, Graduate Text in Mathematics 155, Springer, 1995.

[Kh] V. K. Kharchenko, Connected braided Hopf algebras. (English summary) J. Algebra 307 (2007), no. 1, $24-48$.

[Mj1] S. Majid, Foundations of quantum group theory, Cambridge University Press, 1995.

[NT] C. Năstăsescu, B. Torrecillas, Graded coalgebras, Tsukuba J. Math. 17 (1993), 461-479.

[Ni] Nichols, W. D. Bialgebras of type one. Comm. Algebra 6 (1978), no. 15, 1521-1552.

[Po] N. Popescu, Abelian Categories with Application to Rings and Modules, Academic Press, London \& New York, (1973).

[Ro] M. Rosso, Quantum groups and quantum shuffles, Invent. Math. 133 (1998), no. 2, 399-416.

University of Ferrara, Department of Mathematics, Via Machiavelli 35, Ferrara, I-44121, Italy

E-mail address: alessandro.ardizzoni@unife.it

$U R L:$ http://www.unife.it/utenti/alessandro.ardizzoni

University of Ferrara, Department of Mathematics, Via Machiavelli 35, Ferrara, I-44121, Italy

E-mail address: men@unife.it

URL: http://www.unife.it/utenti/claudia.menini 